\title{
A nonlinear dynamical system on the set of Laguerre entire functions
}

\author{
Yuri Kozitsky ${ }^{a, b, c, 1}$ and Lech Wołowski ${ }^{a, d}$ \\ ${ }^{a}$ Institute of Mathematics, \\ Marie Curie-Skłodowska University, Lublin 20-031 Poland \\ ${ }^{b}$ Institute for Condensed Matter Physics, Lviv 290011 Ukraine \\ ${ }^{c}$ e-mail: jkozi@golem.umcs.lublin.pl \\ ${ }^{d}$ e-mail: lechw@golem.umcs.lublin.pl
}

\begin{abstract}
A nonlinear modification of the Cauchy problem $D_{t} f(t, z)=$ $\theta D_{z} f(t, z)+z D_{z}^{2} f(t, z), t \in \mathbb{R}_{+}=[0,+\infty), z \in \mathbb{C}, \theta \geq 0, f(0, z)=g(z) \in \mathcal{L}$ is considered. The set $\mathcal{L}$ consists of Laguerre entire functions, which one obtains as a closure of the set of polynomials having real nonpositive zeros only in the topology of uniform convergence on compact subsets of $\mathbb{C}$. The modification means that the time half-line $\mathbb{R}_{+}$is divided onto the intervals $\mathcal{I}_{n}=[(n-1) \tau, n \tau], n \in \mathbb{N}, \tau>0$, and on each $\mathcal{I}_{n}$ the evolution is to be described by the above equation but at the endpoints the function $f(t, z)$ is changed: $f(n \tau, z) \rightarrow\left[f\left(n \tau, z \delta^{-1-\lambda}\right)\right]^{\delta}$, with $\lambda>0$ and an integer $\delta \geq 2$. The resolvent operator of such problem preserves the set $\mathcal{L}$. It is shown that for $t \rightarrow+\infty$, the asymptotic properties of $f(t, z)$ change considerably when the parameter $\tau$ reaches a threshold value $\tau_{*}$. The limit theorems for $\tau<\tau_{*}$ and for $\tau=\tau_{*}$ are proven. Certain applications, including limit theorems for weakly and strongly dependent random vectors, are given.
\end{abstract}

Keywords: Holomorphic Operators; Fixed Points; Stability; Convergence; Cauchy Problem

Mathematical Subject Classification: 30D15, 35K55, 58F39

${ }^{1}$ Supported in part under the Grant KBN No 2 P03A 02915 


\section{Setup}

\section{$1.1 \quad$ Introduction}

The Laguerre entire functions [3] are obtained as uniform limits on compact subsets of $\mathbb{C}$ of the sequences of polynomials possessing real nonpositive zeros only. These functions are being studied by many authors during this century in view of their various applications (see also [7]). In [6] the set of Laguerre entire functions $\mathcal{L}$ was described in the framework of locally convex spaces of exponential type entire functions. In particular, it was shown that the Cauchy problem

$$
\begin{aligned}
\frac{\partial f(t, z)}{\partial t} & =\theta \frac{\partial f(t, z)}{\partial z}+z \frac{\partial^{2} f(t, z)}{\partial z^{2}}, \quad t \in \mathbb{R}_{+} \stackrel{\text { def }}{=}[0,+\infty), \quad z \in \mathbb{C}, \\
f(0, z) & =g(z) \in \mathcal{L}, \quad \theta \geq 0 .
\end{aligned}
$$

has a unique solution in $\mathcal{L}$ at least for $t$ small enough. This solution was obtained in an integral form and its possible asymptotic properties when $t \rightarrow+\infty$ were considered. In this paper, a nonlinear modification of this problem is introduced and studied. We divide the time half-line onto the intervals $[(n-1) \tau, n \tau], n \in \mathbb{N}$ with certain $\tau>0$. On each such an interval the evolution is to be described by the above equation but at the endpoints the function $f(t, z)$ is changed

$$
f(n \tau, z) \rightarrow\left[f\left(n \tau, z \delta^{-1-\lambda}\right)\right]^{\delta},
$$

with a fixed $\lambda>0$ and an integer $\delta \geq 2$. For this dynamical system, we construct the evolution operator as a holomorphic nonlinear map between the Fréchet spaces of entire functions, which preserves the set of Laguerre entire functions. Here we use the properties of the operators having the form $\varphi\left(\Delta_{\theta}\right)$ with $\Delta_{\theta}=(\theta+z D) D$ and $\varphi \in \mathcal{L}$ studied in [6]. For $\lambda<1 / 2$, we show that, for sufficiently small values of $\tau$, the asymptotic properties of $f(t, z)$, $t \rightarrow+\infty$ qualitatively are the same as in the case where the evolution is described only by the transformation (1.1). At the same time, it is shown that there exists a threshold value $\tau_{*}>0$ such that the asymptotic behaviour of $f(t, z)$ changes drastically when $\tau$ achieves this value. The description of this phenomenon is based upon the properties of the evolution operator fixed points. The results obtained are then used to describe a similar evolution on the sets of isotropic (i.e. $O(N)$-invariant) analytic functions and measures 
defined on $\mathbb{R}^{N}$. In particular, the limit theorems for strongly and weakly dependent $N$-dimensional random vectors are proved.

Every statement given below in the form of Proposition either was proved in [6] or may be proven in an evident way.

\subsection{Definitions and Main Results}

Let $\mathcal{E}$ be the set of all entire functions $\mathbb{C} \rightarrow \mathbb{C}$. For $b>0$, we define

$$
\mathcal{B}_{b}=\left\{f \in \mathcal{E} \mid\|f\|_{b}<\infty\right\}
$$

where

$$
\|f\|_{b}=\sup _{k \in \mathbb{N}_{0}}\left\{b^{-k}\left|f^{(k)}(0)\right|\right\}, \quad f^{(k)}(0)=\left(D^{k} f\right)(0)=\frac{d^{k} f}{d z^{k}}(0),
$$

and $\mathbb{N}_{0}$ stands for the set of nonnegative integers. For $a \geq 0$, let

$$
\mathcal{A}_{a}=\bigcap_{b>a} \mathcal{B}_{b}=\left\{f \in \mathcal{E} \mid(\forall b>a)\|f\|_{b}<\infty\right\}
$$

Proposition $1.1\left(\mathcal{B}_{b},\|\cdot\|_{b}\right)$ is a Banach space, $\mathcal{A}_{a}$ equipped with the topology defined by the family $\left\{\|\cdot\|_{b}, b>a\right\}$ is a Fréchet space.

An equivalent topology on $\mathcal{A}_{a}$ may be introduced by means of the family $\left\{|\cdot|_{b}, b>a\right\}$ of the norms

$$
|f|_{b} \stackrel{\text { def }}{=} \sup _{z \in \mathbb{C}}\{|f(z)| \exp (-b|z|)\}
$$

Definition 1.1 A family $\mathcal{L}$ is formed by the entire functions possessing the representation

$$
\begin{gathered}
f(z)=C z^{m} \exp (\alpha z) \prod_{j=1}^{\infty}\left(1+\gamma_{j} z\right) \\
C \in \mathbb{C}, m \in \mathbb{N}_{0}, \quad \alpha \geq 0, \quad \gamma_{j} \geq \gamma_{j+1} \geq 0, \sum_{j=1}^{\infty} \gamma_{j}<\infty .
\end{gathered}
$$


The elements of $\mathcal{L}$ are known as the Laguerre entire functions [3]. Due to Laguerre and Pólya (see e.g. [3], [7]), we know that $\mathcal{L}$ consists of the polynomials possessing real nonpositive zeros only as well as of their uniform limits on compact subsets of $\mathbb{C}$. Let $\mathcal{P}_{\mathcal{L}}$ be the set of polynomials belonging to $\mathcal{L}$ and

$$
\begin{gathered}
\mathcal{L}^{+} \stackrel{\text { def }}{=}\{f \in \mathcal{L} \mid f(0)>0\}, \quad \mathcal{L}^{(1)} \stackrel{\text { def }}{=}\{f \in \mathcal{L} \mid f(0)=1\}, \\
\mathcal{L}_{a} \stackrel{\text { def }}{=} \mathcal{L} \cap \mathcal{A}_{a}, \quad \mathcal{L}_{a}^{+} \stackrel{\text { def }}{=} \mathcal{L}^{+} \cap \mathcal{A}_{a}, \quad \mathcal{L}_{a}^{(1)} \stackrel{\text { def }}{=} \mathcal{L}^{(1)} \cap \mathcal{A}_{a} .
\end{gathered}
$$

Given $\theta \geq 0$, a map $\Delta_{\theta}: \mathcal{E} \rightarrow \mathcal{E}$ is defined to be

$$
\left(\Delta_{\theta} f\right)(z)=(\theta+z D) D f(z)=\theta \frac{d f(z)}{d z}+z \frac{d^{2} f(z)}{d z^{2}} .
$$

For $F(z)=f\left(z^{2}\right)$, one observes

$$
\left(\Delta_{\theta} f\right)\left(z^{2}\right)=\frac{1}{4}\left(\frac{2 \theta-1}{z} \frac{d F(z)}{d z}+\frac{d^{2} F(z)}{d z^{2}}\right),
$$

which means that, for $\theta=N / 2, N \in \mathbb{N}$, the map (1.7) is connected with the radial part of the $N$-dimensional Laplacian

$$
\Delta_{r}=\frac{N-1}{r} \frac{\partial}{\partial r}+\frac{\partial^{2}}{\partial r^{2}}
$$

Consider now the Cauchy problem:

$$
\begin{aligned}
\frac{\partial f(t, z)}{\partial t} & =\left(\Delta_{\theta} f\right)(t, z), \quad t \in \mathbb{R}_{+}, z \in \mathbb{C}, \\
f(0, z) & =g(z)
\end{aligned}
$$

and let the initial condition have the form

$$
g(z)=\exp (-\varepsilon z) h(z), \quad h \in \mathcal{A}_{0}, \quad \varepsilon \geq 0 .
$$

The following statement was proven in [6] as Theorem 1.6.

Proposition 1.2 (i) For every $\theta \geq 0$ and $g \in \mathcal{E}$ having the form (1.10), the problem (1.9) has a unique solution in $\mathcal{A}_{\varepsilon}$, which possesses the following integral representation

$$
f(t, z)=\exp \left(-\frac{z}{t}\right) \int_{0}^{+\infty} s^{\theta-1} w_{\theta}\left(\frac{z s}{t}\right) e^{-s} g(t s) d s, \quad t>0,
$$




$$
w_{\theta}(z) \stackrel{\text { def }}{=} \sum_{k=0}^{\infty} \frac{z^{k}}{k ! \Gamma(\theta+k)} .
$$

(ii) If in (1.10) $\varepsilon>0$, the solution (1.11) converges in $\mathcal{A}_{\varepsilon}$ to zero when $t \rightarrow+\infty$.

(iii) If in (1.10) $h \in \mathcal{L}_{0}$ and $\varepsilon=0$, the solution (1.11) also belongs to $\mathcal{L}_{0}$. It diverges when $t \rightarrow+\infty$, which means $M_{f}(t, r) \rightarrow+\infty$ for every $r \in \mathbb{R}_{+}$. Here

$$
M_{f}(t, r) \stackrel{\text { def }}{=} \sup _{|z| \leq r}|f(t, z)| .
$$

By claim (ii), the so called stabilization of solutions holds (see e.g. [4] and |11).

We modify the evolution described by the equation (1.9) as follows. Let us divide the time half-line $\mathbb{R}_{+}$onto the intervals $[(n-1) \tau, n \tau], n \in \mathbb{N}$ with some $\tau>0$. On each such an interval, the evolution is to be described by (1.9) but at the moments $t=n \tau, n \in \mathbb{N}_{0}$ the function is changed as follows

$$
f(n \tau, z) \rightarrow\left[f\left(n \tau, z \delta^{-1-\lambda}\right)\right]^{\delta},
$$

with a fixed $\lambda>0$ and an integer $\delta \geq 2$. It is more convenient to deal with the sequence of functions depending on $t$ from one such interval instead of considering one function with $t$ varying on the sequence of intervals. In what follows, we consider the sequence of functions $\left\{f_{n}(t, z), n \in N_{0}\right\}$, each of which is a solution of the following Cauchy problem

$$
\begin{aligned}
\frac{\partial f_{n}(t, z)}{\partial t} & =\tau\left(\Delta_{\theta} f_{n}\right)(t, z), \quad \tau \geq 0, \quad t \in[0,1], \quad z \in \mathbb{C}, \\
f_{n}(0, z) & =\left[f_{n-1}\left(1, z \delta^{-1-\lambda}\right)\right]^{\delta}, n \in \mathbb{N}, \\
f_{0}(1, z) & =g(z) \in \mathcal{L}^{+} .
\end{aligned}
$$

Any $g \in \mathcal{L}^{+}$is described by the parameters $C, \alpha,\left\{\gamma_{j}\right\}$ (see (1.4) and (1.5)) and one can show that $g \in \mathcal{L}_{\alpha}^{+}$. For such functions, we define

$$
m_{k}(g)=\sum_{j=1}^{\infty} \gamma_{j}^{k}, \quad k \in \mathbb{N},
$$

and

$$
I(g)=\left\{\begin{array}{l}
{\left[0,\left(\delta^{\lambda}-1\right) / \alpha\right], \quad \alpha>0} \\
{[0, \infty), \quad \alpha=0}
\end{array} .\right.
$$


Proposition 1.2 implies the existence of solutions of (1.13) at least for $g \in \mathcal{L}_{0}$. The first our theorem establishes the existence of these solutions for more general situations.

Theorem 1.1 Let $g \in \mathcal{L}^{+}$and $\tau \in I(g)$ be chosen. Then for every $n \in \mathbb{N}$ and $\theta \geq 0$, the problem (1.13) has a unique solution $f_{n}$, which belongs to $\mathcal{L}_{\alpha}^{+}$.

For $\tau=0$, the sequence $\left\{f_{n}\right\}$ can be found explicitly:

$$
f_{n}(t, z)=\left[g\left(z \delta^{-n(1+\lambda)}\right)\right]^{\delta^{n}} .
$$

If $g \in \mathcal{L}^{(1)}$, this sequence converges in $\mathcal{A}_{\alpha}$ to the function $f(t, z) \equiv 1$. Thus one may expect that the same or similar convergence holds also for small positive values of $\tau$. On the other hand, for large values of $\tau$, claim (iii) of Proposition 1.2 suggests the divergence. Our aim in this work is to study the questions: (a) does there exist the intermediate value of $\tau$, say $\tau_{*}$, which separates such "small" and "large" values; (b) what would be the convergence of the sequence $\left\{f_{n}\right\}$ for $\tau=\tau_{*}$. The answer has been found for the values of $\lambda$ restricted to the interval $\lambda \in(0,1 / 2)$ when the initial element $g$ is being chosen in a subset of $\mathcal{L}^{+}$defined by $\lambda$ as follows. Let

$$
\vartheta(\lambda) \stackrel{\text { def }}{=} \frac{1-\delta^{-\epsilon}}{\delta^{\lambda}-\delta^{-\epsilon}}, \quad \epsilon=\frac{1-2 \lambda}{4} .
$$

Definition 1.2 A family $\mathcal{L}(\lambda)$ consists of the functions $g \in \mathcal{L}^{(1)}$ which are not constant and are such that

$$
\frac{m_{2}(g)}{\left[\alpha+m_{1}(g)\right]^{2}} \leq \frac{\delta^{1 / 2}}{\theta+1} \vartheta(\lambda), \quad \frac{m_{2}(g)}{\left[m_{1}(g)\right]^{2}} \leq \frac{\delta^{1 / 2}}{\theta+1} .
$$

Thereby, we state our main theorem.

Theorem 1.2 For every $\theta \geq 0$ and $g \in \mathcal{L}(\lambda)$, there exist a positive $\tau_{*} \in I(g)$ and a function $C:\left[0, \tau_{*}\right] \rightarrow \mathbb{R}_{+}$such that

(i) for $\tau<\tau_{*}$, the sequence of solutions of (1.19)

$\left\{f_{n}(t, z) \mid n \in \mathbb{N}_{0}, f_{0}(1, z)=C(\tau) g(z)\right\}$ converges

in $\mathcal{A}_{\beta_{*}^{-1}}, \beta_{*} \stackrel{\text { def }}{=} \tau_{*} /\left(\delta^{\lambda}-1\right)$ to the function $f(t, z) \equiv 1$;

(ii) for $\tau=\tau_{*}$, the sequence $\left\{f_{n}(t, z) \mid n \in \mathbb{N}_{0}, f_{0}(1, z)=C\left(\tau_{*}\right) g(z)\right\}$ converges in $\mathcal{A}_{\beta_{*}^{-1}}$, to

$$
f_{*}(t, z)=\delta^{-\delta \theta \lambda /(\delta-1)}\left[1-t\left(1-\delta^{-\lambda}\right)\right]^{-\theta} \exp \left(\frac{1}{\tau_{*}} \frac{1-\delta^{-\lambda}}{1-t\left(1-\delta^{-\lambda}\right)} z\right) .
$$


Remark 1.1 The convergence to nontrivial (neither zero nor infinity) limits needs to control the constant $C$ in the representation (1.4) of the initial element of $\left\{f_{n}\right\}$. Otherwise one obtains only such trivial limits for "small" and "large" values of this constant.

\subsection{Some Applications and Further Results}

Let $\mathcal{E}^{(N)}, N \in \mathbb{N}$ be the set of analytic functions $F: \mathbb{R}^{N} \rightarrow \mathbb{C}$. For appropriate $F \in \mathcal{E}^{(N)}$ and some $b>0$, we set

$$
\|F\|_{b, N} \stackrel{\text { def }}{=} \sup _{x \in \mathbb{R}^{N}}\left\{|F(x)| \exp \left(-b|x|^{2}\right)\right\},
$$

where $|x|$ is the Euclidean norm of $x \in \mathbb{R}^{N}$. Let

$$
\mathcal{A}_{a}^{(N)} \stackrel{\text { def }}{=}\left\{F \in \mathcal{E}^{(N)} \mid\|F\|_{b, N}<\infty, \forall b>a\right\}, \quad a \geq 0 .
$$

This set equipped with the topology generated by the family $\left\{\|\cdot\|_{b, N}, b>a\right\}$ becomes a Fréchet space. Let $O(N)$ stand for the group of all orthogonal transformations of $\mathbb{R}^{N}$. A function $F \in \mathcal{E}^{(N)}$ is said to be isotropic if for every $U \in O(N)$ and all $x \in \mathbb{R}^{N}, F(U x)=F(x)$. The subset of $\mathcal{E}^{(N)}$ consisting of isotropic functions is denoted by $\mathcal{E}_{\text {isot }}^{(N)}$. Now let $\mathcal{P}_{\text {isot }}^{(N)} \subset \mathcal{E}_{\text {isot }}^{(N)}$ stand for the set of isotropic polynomials. The classical Study-Weyl theorem [9] (see also [Q] implies that there exists a bijection between the set of all polynomials of one complex variable $\mathcal{P}$ and $\mathcal{P}_{\text {isot }}^{(N)}$ established by

$$
\mathcal{P}_{\text {isot }}^{(N)} \ni P(x)=p((x, x)) \in \mathcal{P},
$$

where (.,.) is the scalar product in $\mathbb{R}^{N}$. Obviously each a function $F$ having the form

$$
F(x)=f((x, x)),
$$

with certain $f \in \mathcal{E}$, belongs to $\mathcal{E}_{\text {isot }}^{(N)}$. Given a subset $\mathcal{X} \subset \mathcal{E}$, we write $\mathcal{X}\left(\mathbb{R}^{N}\right)$ for the subset of $\mathcal{E}_{\text {isot }}^{(N)}$ consisting of the functions obeying (1.22) with $f \in \mathcal{X}$. In this notation $\mathcal{P}_{\text {isot }}^{(N)}=\mathcal{P}\left(\mathbb{R}^{N}\right)$. Consider a map

$$
\mathcal{E}_{\text {isot }}^{(N)} \ni F \mapsto\left(\Delta+\frac{d}{(x, x)}(x, \nabla)\right) F \in \mathcal{E}_{\text {isot }}^{(N)},
$$


where $\Delta$ and $\nabla$ stand for the Laplacian and for the gradient in $\mathbb{R}^{N}$. For a pair of functions $F$ and $f$ satisfying (1.22), one has (c.f. (1.8))

$$
\left(\Delta+\frac{d}{(x, x)}(x, \nabla)\right) F(x)=4\left(\Delta_{\theta} f\right)((x, x)),
$$

where $\Delta_{\theta}$ is defined by $(1.7)$ with

$$
\theta=\frac{N+d}{2}
$$

Now let us consider the following Cauchy problem - an analog of (1.13):

$$
\begin{aligned}
\frac{\partial F_{n}(t, x)}{\partial t} & =\tau\left(\Delta+\frac{d}{(x, x)}(x, \nabla)\right) F_{n}(t, x), t \in[0,1], x \in \mathbb{R}^{N}, \\
F_{n}(0, x) & =\left[F_{n-1}\left(1, x \delta^{-(1+\lambda) / 2}\right)\right]^{\delta}, n \in \mathbb{N}, \\
F_{0}(1, x) & =G(x) \in \mathcal{L}^{+}\left(\mathbb{R}^{N}\right) .
\end{aligned}
$$

For $G \in \mathcal{L}^{+}\left(\mathbb{R}^{N}\right)$, there exists $g \in \mathcal{L}^{+}$such that $G$ and $g$ satisfy (1.22), thus the interval (1.15) is defined for such $G$. The direct corollary of Theorem 1.1 reads

Theorem 1.3 For every $d \geq-N, G \in \mathcal{L}^{+}\left(\mathbb{R}^{N}\right), \tau \in I(g)$, and $n \in \mathbb{N}$, the problem (1.25) has a unique solution $F_{n}$, which also belongs to $\mathcal{L}^{+}\left(\mathbb{R}^{N}\right)$.

For $\lambda \in(0,1 / 2)$, we have an analog of Theorem 1.2 .

Theorem 1.4 For every $d \geq-N$ and $g \in \mathcal{L}(\lambda)$, there exist a positive $\tau_{*} \in$ $I(g)$ and $C:\left[0, \tau_{*}\right] \rightarrow \mathbb{R}_{+}$, such that

(i) for $\tau<\tau_{*}$, the sequence of solutions of (1.25) $\left\{F_{n}(t, x) \mid n \in \mathbb{N}_{0}, \quad F_{0}(1, z)=C(\tau) g((x, x))\right\}$ converges in $\mathcal{A}_{\beta_{*}^{-1}}^{(N)}, \beta_{*} \stackrel{\text { def }}{=} \tau_{*} /\left(\delta^{\lambda}-1\right)$ to the function $F(t, x) \equiv 1$;

(ii) $\quad$ for $\tau=\tau_{*},\left\{F_{n}(t, x) \mid n \in \mathbb{N}_{0}, \quad F_{0}(1, x)=C\left(\tau_{*}\right) g((x, x))\right\}$ converges in $\mathcal{A}_{\beta_{*}^{-1}}^{(N)}$ to

$$
F_{*}(t, x)=\delta^{-\delta \theta \lambda /(\delta-1)}\left[1-t\left(1-\delta^{-\lambda}\right)\right]^{-\theta} \exp \left(\frac{1}{\tau_{*}} \frac{1-\delta^{-\lambda}}{1-t\left(1-\delta^{-\lambda}\right)}(x, x)\right),
$$

where $\theta$ is given by (1.24). 
Let $\mathcal{M}$ stand for the set of probability measures $\mu$ on $\mathbb{R}^{N}$ such that

$$
\int_{\mathbb{R}^{N}} \exp (\varepsilon(x, x)) \mu(d x)<\infty
$$

with certain $\varepsilon>0$. For each such a measure, the function

$$
F_{\mu}(x) \stackrel{\text { def }}{=} \int_{\mathbb{R}^{N}} \exp ((x, y)) \mu(d y),
$$

belongs to $\mathcal{E}^{(N)}$. For a Borel subset $B \subset \mathbb{R}^{N}$, we let

$B-x=\left\{y \in \mathbb{R}^{N} \mid x+y \in B\right\}, U B=\left\{x \in \mathbb{R}^{N} \mid U^{-1} x \in B\right\}, U \in O(N)$.

A measure $\mu \in \mathcal{M}$ is said to be isotropic if it is $O(N)$-invariant (i.e. $\mu(U B)=$ $\mu(B))$, the subset $\mathcal{M}_{\text {isot }} \subset \mathcal{M}$ is to consist of such isotropic measures. Obviously, $F_{\mu} \in \mathcal{E}_{\text {isot }}^{(N)}$ for $\mu \in \mathcal{M}_{\text {isot. }}$. Now let $\mathcal{M}\left(\mathbb{R}^{N}\right)$ be the subset of $\mathcal{M}_{\text {isot }}$ consisting of the measures for which $F_{\mu} \in \mathcal{L}^{(1)}\left(\mathbb{R}^{N}\right)$. For a pair of measures $\mu, \nu \in \mathcal{M}$, their convolution is as usual

$$
(\mu \star \nu)(B)=\int_{\mathbb{R}^{N}} \mu(B-x) \nu(d x) .
$$

Since $F_{\mu \star \nu}=F_{\mu} F_{\nu}$, the measure $\mu \star \nu$ belongs to $\mathcal{M}\left(\mathbb{R}^{N}\right)$ whenever $\mu$ and $\nu$ possess this property. Now let $\delta, \lambda$, and $\tau$ be as in (1.13), (1.25). Consider the sequence $\left\{\mu_{n}, n \in \mathbb{N}_{0}\right\}$ defined

$$
\mu_{n}(d y)=\frac{1}{M_{n}(\tau)} \exp (\tau(y, y)) \mu_{n-1}^{\star \delta}\left(\delta^{(1+\lambda) / 2} d y\right), \quad \mu_{0}=\nu \in \mathcal{M}\left(\mathbb{R}^{N}\right)
$$

where

$$
M_{n}(\tau) \stackrel{\text { def }}{=} \int_{\mathbb{R}^{N}} \exp (\tau(y, y)) \mu_{n-1}^{\star \delta}\left(\delta^{(1+\lambda) / 2} d y\right),
$$

and $\mu^{\star \delta}$ is the convolution of $\delta$ copies of $\mu$. The measure $\mu_{n-1}^{\star \delta}\left(\delta^{(1+\lambda) / 2}\right.$. $)$ describes the probability distribution of the normalized sum of $\delta$ identically distributed independent random vectors. By means of the multiplier $\exp (\tau(y, y))$ in (1.29), we set these vectors being dependent, thus the measure $\mu_{n}$ describes the probability distribution of the following random vector

$$
X^{(n)}=\frac{1}{\sqrt{\delta}} \delta^{-\lambda / 2}\left(X_{1}^{(n-1)}+\ldots+X_{\delta}^{(n-1)}\right) .
$$


The normalization of this sum is "abnormal" (more than normal) due to the additional factor $\delta^{-\lambda / 2}$. Every $X^{(m)}$ is the sum of $\delta^{m}$ vectors of the zero level. Such random vectors are known to be hierarchically dependent (see e.g. [5]). Their dependence is proportional to the parameter $\tau$ - it disappears if $\tau=0$. Therefore, one may expect that, for small positive values of $\tau$, the dependence remains weak and the vectors obey the classical central limit theorem. In this case, due to the factors $\delta^{-\lambda / 2}$, the sequence of measures $\left\{\mu_{n}\right\}$ ought to be asymptotically degenerate at zero, which means that the corresponding by (1.27) sequence $\left\{F_{\mu_{n}}\right\}$ converges to the function $F(x) \equiv 1$. But the functions $F_{\mu_{n}}$ may be obtained as solutions of the problem (1.25). To use this fact we construct the subset of $\mathcal{M}\left(\mathbb{R}^{N}\right)$ corresponding to $\mathcal{L}(\lambda)$ introduced by Definition 1.2. Choose $\lambda \in(0,1 / 2)$. For a measure $\nu \in \mathcal{M}\left(\mathbb{R}^{N}\right)$, let $g \in \mathcal{L}^{(1)}$ be the function such that $F_{\nu}(x)=g((x, x))$. Then

$$
\mathcal{M}_{\lambda}\left(\mathbb{R}^{N}\right) \stackrel{\text { def }}{=}\left\{\nu \in \mathcal{M}\left(\mathbb{R}^{N}\right) \mid g \in \mathcal{L}(\lambda)\right\} .
$$

The following assertion is a corollary of Theorem 1.4 for $d=0$.

Theorem 1.5 For every $N \in \mathbb{N}$ and $\nu \in \mathcal{M}_{\lambda}\left(\mathbb{R}^{N}\right)$, there exists $\tau_{*}>0$ such that

(i) for $\tau<\tau_{*}$, the sequence of measures defined by (1.29) $\left\{\mu_{n} \mid n \in \mathbb{N}_{0}, \mu_{0}=\nu\right\}$ converges weakly to the measure degenerate at zero;

(ii) for $\tau=\tau_{*}$, this sequence converges weakly to the isotropic Gaussian measure with variance $2 N\left(\delta^{\lambda}-1\right) / \tau_{*}$.

It should be pointed out that the convergence to a nondegenerate measure for the abnormal normalization described by claim (ii) means the appearance of a strong dependence between the random vectors considered. For $\tau<\tau_{*}$, the dependence is weak and the classical central limit theorem ought to hold. To show this we introduce the classical normalization instead of (1.30). So we set along with (1.29):

$$
\begin{aligned}
\tilde{\mu}_{n}(d y) & =\frac{1}{\tilde{M}_{n}(\tau)} \exp \left(\delta^{-n \lambda / 2} \tau(y, y)\right) \tilde{\mu}_{n-1}^{\star \delta}(\sqrt{\delta} d y), \mu_{0}=\nu \in \mathcal{M}\left(\mathbb{R}^{N}\right) \\
\tilde{M}_{n}(\tau) & =\int_{\mathbb{R}^{N}} \exp \left(\delta^{-n \lambda / 2} \tau(y, y)\right) \tilde{\mu}_{n-1}^{\star \delta}(\sqrt{\delta} d y) .
\end{aligned}
$$


Theorem 1.6 Let $N, \nu$, and $\tau_{*}$ be as in Theorem 1.5. Then for $\tau<\tau_{*}$, the sequence of measures $\left\{\tilde{\mu}_{n} \mid n \in \mathbb{N}_{0}, \tilde{\mu}_{0}=\nu\right\}$ defined by (1.32) converges weakly to an isotropic Gaussian measure.

\section{Preliminaries}

\subsection{Laguerre Entire Functions and Evolution Operator}

We start with the description of the Fréchet spaces $\mathcal{A}_{a}$. A subset $B \subset \mathcal{A}_{a}$ is said to be bounded in $\mathcal{A}_{a}$ if for every $b>a$, there exists $K_{b}>0$ such that, for all $f \in B,\|f\|_{b} \leq K_{b}$.

Proposition 2.1 For every $a \geq 0$, the space $\mathcal{A}_{a}$ possesses the properties:

(i) the relative topology on bounded subsets of $\mathcal{A}_{a}$ coincides with the topology of uniform convergence on compact subsets of $\mathbb{C}$;

(ii) multiplication, i.e., $(f, g) \mapsto f g$ is a continuous map from $\mathcal{A}_{a} \times \mathcal{A}_{b}$ into $\mathcal{A}_{a+b}$

(iii) differentiation, i.e. $f \mapsto f^{\prime}$ is a continuous self-map of $\mathcal{A}_{a}$.

Remark 2.1 It can be easily shown that, for positive $a$ and $b$,

$$
\|f g\|_{a+b} \leq\|f\|_{a}\|g\|_{b},
$$

thus $(f, g) \mapsto f g$ is a continuous map from $\mathcal{B}_{a} \times \mathcal{B}_{b}$ into $\mathcal{B}_{a+b}$, which implies claim (ii) of the latter statement.

Proposition 2.2 Every sequence $\left\{f_{n}, n \in \mathbb{N}\right\} \subset \mathcal{L}_{a}, a \geq 0$, that converges in $\mathcal{E}$ to a function $f \in \mathcal{A}_{a}$, which does not vanish identically, is a bounded subset of $\mathcal{A}_{a}$ and hence, by claim (i) of Proposition 2.1, it converges in $\mathcal{A}_{a}$ to $f \in \mathcal{L}$.

For $f \in \mathcal{L}^{+}$, one has $f(0)>0$ (see (1.5)). Therefore, for such a function, there exists the neighborhood $\mathcal{D}$ of the origin in which $f \neq 0$, hence the following holomorphic function can be defined

$$
\varphi(z)=\log f(z), \quad z \in \mathcal{D} .
$$

In the sequel we use the notation

$$
\varphi^{(k)}=\left(D^{k} \log f\right)(0), \quad k \in \mathbb{N}_{0} .
$$


Proposition 2.3 [The sign rule] Let $f \in \mathcal{L}^{+}$, then

$$
(-1)^{k-1} \varphi^{(k)} \geq 0, \quad k \in \mathbb{N} .
$$

Equalities hold simultaneously for all $k \geq 2$ and only for $f(z)=C \exp (a z)$.

Lemma 2.1 For a sequence $\left\{f_{n}(z) \mid n \in \mathbb{N}_{0} f_{n} \in \mathcal{L}^{+}\right\}$, let the derivatives (2.3) satisfy:(i) $\left\{\varphi_{n}^{(k)}\right\}$ converges to $\varphi^{(k)}, k=0,1$; (ii) $\left\{\varphi_{n}^{(2)}\right\}$ converges to zero. Then $\left\{f_{n}\right\}$ converges to $\exp \left(\varphi^{(0)}+\varphi^{(1)} z\right)$ in $\mathcal{A}_{\psi}, \psi=\sup \varphi_{n}^{(1)}$.

Proof. By claim (ii) of Proposition 2.1 and Proposition 2.2, to prove this statement we only need to show that the sequence $\left\{f_{n}(z) / f_{n}(0)\right\}$ converges to $\exp \left(\varphi^{(1)} z\right)$ uniformly on compact subsets of $\mathbb{C}$. Due to known Vitali's theorem and to the fact that, for the functions considered, $M_{f}(r)=f(r)$, we may do this by proving the pointwise convergence of $\left\{f_{n}(z) / f_{n}(0)\right\}$ on $\mathbb{R}_{+}$. To this end we use the specific form of $f \in \mathcal{L}^{(1)}$ given by (1.4). For each $\gamma \geq 0$, one has $\exp \left(\gamma-\frac{1}{2} \gamma^{2}\right) \leq 1+\gamma \leq \exp (\gamma)$. Hence for $z \in \mathbb{R}_{+}$,

$$
\exp \left(z \varphi_{n}^{(1)}+\frac{1}{2} z^{2} \varphi_{n}^{(2)}\right) \leq \frac{f_{n}(z)}{f_{n}(0)} \leq \exp \left(z \varphi_{n}^{(1)}\right),
$$

which yields the stated convergence.

For an entire function $f \in \mathcal{E}$ and $t \geq 0$, we define

$$
\left(\exp \left(t \Delta_{\theta}\right) f\right)(z)=\sum_{k=0}^{\infty} \frac{t^{k}}{k !}\left(\Delta_{\theta}^{k} f\right)(z) .
$$

Proposition 2.4 For every positive $a$ and $t$ obeying at $<1$, and $\theta \geq 0$,

$$
\left\|\exp \left(t \Delta_{\theta}\right) f\right\|_{b} \leq(1-a t)^{-\theta}\|f\|_{a}, \quad b=a /(1-a t),
$$

which means that (2.6) defines a continuous linear map

$$
\mathcal{A}_{a} \ni f \mapsto f_{t} \stackrel{\text { def }}{=}\left(\exp \left(t \Delta_{\theta}\right) f\right) \in \mathcal{A}_{b}, \quad b=a /(1-a t) .
$$

Corollary 2.1 For every positive $a$ and $t_{0}$, a map $\left(0, t_{0}\right) \ni t \mapsto f_{t} \in \mathcal{A}_{b_{0}}$, where $b_{0} \stackrel{\text { def }}{=} a /\left(1-a t_{0}\right)$ and $f_{t}$ is defined by (2.X), is differentiable on $\left(0, t_{0}\right)$ and

$$
\frac{\partial f_{t}}{\partial t}=\Delta_{\theta} f_{t}, \quad t \in\left(0, t_{0}\right) .
$$


One of the main results of [6] is Theorem 1.3 which asserts that the operators of the type of (2.6) preserves the class $\mathcal{L}$. In our case it reads as follows

Proposition 2.5 Let $a, b, t$, and $\theta$ be as in Proposition 2.4. Then the operator (2.0), (2.7) maps $\mathcal{L}_{a}$ into $\mathcal{L}_{b}$.

The following statements have also been proven in [6].

Proposition 2.6 For $t>0$, the above operator has the integral form:

$$
\left(\exp \left(t \Delta_{\theta}\right) f\right)(z)=\exp \left(-\frac{z}{t}\right) \int_{0}^{+\infty} s^{\theta-1} w_{\theta}\left(\frac{z s}{t}\right) e^{-s} f(t s) d s
$$

where $w_{\theta}$ is defined by (1.19).

Remark 2.2 Setting in (2.9) $z=0$, one obtains for $f \in \mathcal{L}^{+}$and $\theta>0$ : $\left(\exp \left(t \Delta_{\theta}\right) f\right)(0)>0$. On the other hand, one has from (2.0)

$$
\left(\exp \left(t \Delta_{\theta}\right) f\right)(0)=\sum_{k=0}^{\infty} \frac{t^{k}}{k !} f^{(k)}(0) \frac{\Gamma(\theta+k)}{\Gamma(\theta)} .
$$

Passing here to the limit $\theta \rightarrow 0$ one gets

$$
\left(\exp \left(t \Delta_{0}\right) f\right)(0)=f(0)>0
$$

Below the case $\theta=0$ is always understood as the above limit.

Proposition 2.7 Let $v \in \mathbb{R}$ and $\exp (v z) h(z) \in \mathcal{A}_{b},(b \geq 0)$. For any $u \geq 0$ obeying the condition $u b<1$,

$$
\exp \left(u \Delta_{\theta}\right) \exp (v z) h(z)=\exp \left(\frac{v z}{1-u v}\right) h_{u}(z)
$$

where

$$
h_{u}(z)=(1-u v)^{-\theta} \exp \left(u(1-u v) \Delta_{\theta}\right) h\left(\frac{z}{(1-u v)^{2}}\right) .
$$

Moreover, if $h \in \mathcal{A}_{a}$, then $h_{u} \in \mathcal{A}_{c}$, where

$$
c=a(1-u v)^{-1}(1-(v+a) u)^{-1} .
$$


By means of (2.6), we construct the evolution operator which solves (1.13):

$$
f_{n}(t, z)=\exp \left(t \tau \Delta_{\theta}\right)\left[f_{n-1}\left(1, z \delta^{-1-\lambda}\right)\right]^{\delta} \stackrel{\text { def }}{=} T_{t}\left(f_{n-1}(t, \cdot)\right)(z),
$$

provided all $f_{n}(t, z)$ belong to the domain of $T_{t}, t \in[0,1]$. For short we write

$$
f_{n}(1, z) \stackrel{\text { def }}{=} f_{n}(z), \quad T_{1} \stackrel{\text { def }}{=} T .
$$

Thus one has

$$
f_{n}=T\left(f_{n-1}\right) .
$$

We use such $T_{t}$ to define the operators between the Fréchet spaces $\mathcal{A}_{a}$ and the Banach spaces $\mathcal{B}_{b}$. In all such cases we denote them by $T_{t}$ pointing out if necessary between which spaces acts given $T_{t}$. Combining claim (ii) of Proposition 2.1 with Propositions 2.4 and 2.5 one has

Proposition 2.8 For every $a<\delta^{\lambda} / t \tau$, the operator $T_{t}$ continuously maps: $\mathcal{B}_{a} \rightarrow \mathcal{B}_{b}, \mathcal{A}_{a} \rightarrow \mathcal{A}_{b}$, and $\mathcal{L}_{a} \rightarrow \mathcal{L}_{b}$, where $b=a \delta^{-\lambda} /\left(1-a t \tau \delta^{-\lambda}\right)$.

Proposition 2.9 Let the sequence $\left\{f_{n}(z) \mid n \in \mathbb{N}_{0}, f_{0}(z)=g(z) \in \mathcal{L}^{+}\right\}$ defined by (2.17) converge in $\mathcal{A}_{a}, a \geq 0$ to a function $f$. Then the sequence of solutions of (1.13) $\left\{f_{n}(t, z) \mid n \in \mathbb{N}_{0}, f_{0}(z)=g(z)\right\}$, defined by (2.15), converges in $\mathcal{A}_{a}$ to the function

$$
f(t, z)=\left(T_{t} f\right)(z) .
$$

To establish the existence and convergence of $\left\{f_{n}\right\}$ we use an analog of the Fréchet derivative of $T$ and then study the fixed points of $T$ and their stability. The following corollary of Proposition 2.8 allows to define the differentiability of $T$ acting between the Fréchet spaces. For $a \in\left[0, \tau \delta^{-\lambda}\right)$, we set

$$
b(a) \stackrel{\text { def }}{=} \frac{a \delta^{-\lambda}}{1-a \tau \delta^{-\lambda}} .
$$

Corollary 2.2 Let $a<\delta^{\lambda} / \tau$, then there exists $\varepsilon>0$ such that, for all $a^{\prime} \in(a, a+\varepsilon)$, the operator $T$ continuously maps $\mathcal{B}_{a^{\prime}}$ into $\mathcal{B}_{b\left(a^{\prime}\right)}$. 
Definition 2.1 The operator $T: \mathcal{A}_{a} \rightarrow \mathcal{A}_{b(a)}$ is said to be differentiable on $\mathcal{A}_{a}$ if for every $f \in \mathcal{A}_{a}$, there exist $\varepsilon>0$ and a continuous linear operator $T^{\prime}[f]: \mathcal{A}_{a} \rightarrow \mathcal{A}_{b(a)}$ such that, for every $a^{\prime} \in(a, a+\varepsilon), T^{\prime}[f]$ is the Fréchet derivative of $T$ considered as an operator between the Banach spaces $\mathcal{B}_{a^{\prime}}$ and $\mathcal{B}_{b\left(a^{\prime}\right)}$. The operator $T^{\prime}[f]$ is said to be a derivative of $T$ at $f$.

Lemma 2.2 For $a<\delta^{\lambda} / \tau$, the operator $T: \mathcal{A}_{a} \rightarrow \mathcal{A}_{b(a)}$ is differentiable on $\mathcal{A}_{a}$ and its derivative $T^{\prime}[f]$ is the following operator

$$
T^{\prime}[f](h)(z)=\delta \exp \left(\tau \Delta_{\theta}\right)\left(\left(f^{\delta-1} h\right)\left(\delta^{-1-\lambda} z\right)\right) .
$$

Proof. For $a^{\prime} \in\left(a, \delta^{\lambda} / \tau\right)$ and $f, h \in \mathcal{B}_{a^{\prime}}$, one has

$$
\begin{gathered}
T(f+h)=T(f)+\delta \exp \left(\tau \Delta_{\theta}\right)\left(\left(f^{\delta-1} h\right)\left(\delta^{-1-\lambda} \cdot\right)\right)+R(f, h), \\
R(f, h)=\exp \left(\tau \Delta_{\theta}\right)\left(\sum_{k=2}^{\delta}\left(\begin{array}{l}
\delta \\
k
\end{array}\right) f^{\delta-k} h^{k}\right)\left(\delta^{-1-\lambda} \cdot\right) .
\end{gathered}
$$

By means of Remark 2.1, (2.1), and Proposition 2.4, one obtains

$$
\begin{gathered}
\left\|\exp \left(\tau \Delta_{\theta}\right)\left(f^{\delta-k} h^{k}\right)\left(\delta^{-1-\lambda} \cdot\right)\right\|_{b\left(a^{\prime}\right)} \leq \\
\quad\left(1-a^{\prime} \tau \delta^{-\lambda}\right)^{-\theta}\|f\|_{a^{\prime}}^{\delta-k}\|h\|_{a^{\prime}}^{k}, \\
k=1,2, \ldots, \delta
\end{gathered}
$$

This gives for all $a^{\prime} \in\left(a, \delta^{\lambda} / \tau\right)$,

$$
\|R(f, g)\|_{b\left(a^{\prime}\right)}=o\left(\|h\|_{a^{\prime}}\right),
$$

and also for $T^{\prime}$ defined by (2.20),

$$
\left\|T^{\prime}[f](h)\right\|_{b\left(a^{\prime}\right)} \leq \delta\left(1-a^{\prime} \tau \delta^{-\lambda}\right)^{-\theta}\|f\|_{a^{\prime}}^{\delta-1}\|h\|_{a^{\prime}} .
$$

By the latter estimate, $T^{\prime}[f]$ continuously maps $\mathcal{B}_{a^{\prime}}$ into $\mathcal{B}_{b\left(a^{\prime}\right)}$ whereas by the former one, this operator is the Fréchet derivative of $T: \mathcal{B}_{a^{\prime}} \rightarrow \mathcal{B}_{b\left(a^{\prime}\right)}$.

The case of $\tau=0$ was considered in (1.16), thus from now on we assume $\tau>0$. It turns out that it is convenient to remove the explicit dependence on $\tau$ from the operator $T$. To this end we set

$$
\tau \stackrel{\text { def }}{=} \beta\left(\delta^{\lambda}-1\right)
$$


and include $\beta$ into $z$. Then we consider the sequence $\left\{g_{n}(z)\right\}$

$$
\begin{aligned}
g_{n}(z) & =Q\left(g_{n-1}\right)(z), \quad n \in \mathbb{N}, \\
& \stackrel{\text { def }}{=} \exp \left(\left(\delta^{\lambda}-1\right) \Delta_{\theta}\right)\left[g_{n-1}\left(\delta^{-1-\lambda} z\right)\right]^{\delta}, g_{0}(z)=g(\beta z),
\end{aligned}
$$

where $g$ is the function which defines the starting element of $\left\{f_{n}\right\}$. To prove Theorem 1.6 we shall also use the sequence of functions from $\mathcal{L}^{(1)},\left\{\tilde{g}_{n}(z) \mid n \in\right.$ $\left.\mathbb{N}_{0}, \quad \tilde{g}_{0}(z)=g(\beta z)\right\}$, where $g$ is as above, and

$$
\begin{aligned}
\tilde{g}_{n}(z) & =\tilde{Q}_{n}\left(\tilde{g}_{n}\right)(z) \\
& \stackrel{\text { def }}{=} \frac{1}{\tilde{Y}_{n}}\left\{\exp \left(\delta^{-n \lambda}\left(\delta^{\lambda}-1\right) \Delta_{\theta}\right)\left[\tilde{g}_{n-1}\left(\delta^{-1} \cdot\right)\right]^{\delta}\right\}(z), \\
\tilde{Y}_{n} & \stackrel{\text { def }}{=}\left\{\exp \left(\delta^{-n \lambda}\left(\delta^{\lambda}-1\right) \Delta_{\theta}\right)\left[\tilde{g}_{n-1}\left(\delta^{-1} \cdot\right)\right]^{\delta}\right\}(0) .
\end{aligned}
$$

Comparing (2.15), 2.16) with (2.22) one obtains from Proposition 2.8 and Lemma 2.2.

Proposition 2.10 For every $a<\delta^{\lambda} /\left(\delta^{\lambda}-1\right), Q$ is a differentiable (and hence continuous) operator, which maps: $\mathcal{A}_{a} \rightarrow \mathcal{A}_{b^{\prime}}, \mathcal{L}_{a}^{+} \rightarrow \mathcal{L}_{b^{\prime}}^{+}$, where $b^{\prime}=$ $a\left[\delta^{\lambda}-a\left(\delta^{\lambda}-1\right)\right]^{-1}$. Its derivative is

$$
Q^{\prime}[g](h)(z)=\exp \left(\left(\delta^{\lambda}-1\right) \Delta_{\theta}\right)\left(\left[g^{\delta-1} h\right]\left(\delta^{-1-\lambda} z\right)\right) .
$$

For $\tau \in I(g), \beta$ varies in $J(g) \stackrel{\text { def }}{=}(0,1 / \alpha]$ (see 2.21$)$ and $(1.15))$. Let $g \in \mathcal{L}^{+}$ be chosen. Then it possesses the representation (1.4) with $\alpha \geq 0$, which determines the intervals $I(g)$ (1.15) and $J(g)$, and $g \in \mathcal{L}_{\alpha}^{+} \subset \mathcal{A}_{\alpha}$.

Lemma 2.3 For $\tau \in I(g)$, all the elements of $\left\{f_{n} \mid n \in \mathbb{N}_{0}, f_{0}=g\right\}$ belong to $\mathcal{L}_{\alpha}^{+} \subset \mathcal{A}_{\alpha}$, all the elements of $\left\{g_{n} \mid n \in N_{0}, g_{0}(z)=g(\beta z)\right\}$ belong to $\mathcal{L}_{\beta \alpha}^{+}$.

Proof. For $\tau \in I(g), \alpha \leq\left(\delta^{\lambda}-1\right) / \tau<\delta^{\lambda} / \tau$, thus by Corollary 2.2, $T$ maps $\mathcal{A}_{\alpha}$ into $\mathcal{A}_{b(\alpha)}$ with

$$
b(\alpha)=\frac{\alpha \delta^{-\lambda}}{1-\alpha \tau \delta^{-\lambda}} \leq \frac{\alpha \delta^{-\lambda}}{1-1+\delta^{-\lambda}}=\alpha,
$$

which means $T: \mathcal{A}_{\alpha} \rightarrow \mathcal{A}_{\alpha}$. By Proposition 2.8, $T$ maps $\mathcal{L}$ into itself; by Remark 2.2, $(T f)(0)>0$ whenever $f(0)>0$. The second part of Lemma concerning $\left\{g_{n}\right\}$ directly follows from the first one.

Since the starting element of $\left\{g_{n}\right\}$ is of the form $g_{0}(z)=g(\beta z)$, all its elements depend on $\beta$. Therefore, one may consider $g_{n}$ as a map from $J(g)$ into $\mathcal{A}_{1}$. To emphasize this fact we write sometimes $g_{n}(\cdot, \beta)$ instead of $g_{n}$. 
Lemma 2.4 For every $n \in \mathbb{N}_{0}$, the map

$$
g_{n}: J(g) \rightarrow \mathcal{A}_{1}
$$

is differentiable on $\operatorname{Int} J(g)$, its derivative at $\beta$ is an entire function $\dot{g}_{n} \in \mathcal{A}_{1}$.

Proof. Let us show that, for $\beta \in \operatorname{Int} J(g)$, there exists an entire function $\dot{g}_{n} \in \mathcal{A}_{1}$ such that, for $\tilde{\beta} \in \operatorname{Int} J(g)$,

$$
g_{n}(\cdot, \tilde{\beta})-g_{n}(\cdot, \beta)=\Delta \beta \dot{g}_{n}+r_{n}(\cdot, \Delta \beta), \quad \Delta \beta=\tilde{\beta}-\beta,
$$

where $r_{n}(\cdot, \Delta \beta) / \Delta \beta \rightarrow 0$ in $\mathcal{A}_{1}$ when $\Delta \beta \rightarrow 0$. By claim (iii) of Proposition 2.1, differentiation is a continuous self-map of $\mathcal{A}_{a}$. Since $g_{0}(z, \beta)=g(\beta z)$, the stated property obviously holds for $n=0$. For some $n \geq 1$, let $\dot{g}_{n-1}$ obey (2.26) and belong to $\mathcal{A}_{1}$. Then

$$
\begin{aligned}
& g_{n}(\cdot, \tilde{\beta})-g_{n}(\cdot, \beta)=Q\left(g_{n-1}(\cdot, \tilde{\beta})\right)-Q\left(g_{n-1}(\cdot, \beta)\right) \\
= & Q\left[\left(g_{n-1}\right)(\cdot, \beta)+\Delta \beta \dot{g}_{n-1}+r_{n-1}(\cdot, \Delta \beta)\right]-Q\left(g_{n-1}(\cdot, \beta)\right) .
\end{aligned}
$$

By means of the derivative (2.24), it can be written as

$$
g_{n}(\cdot, \tilde{\beta})-g_{n}(\cdot, \beta)=\Delta \beta Q^{\prime}\left[g_{n-1}\right]\left(\dot{g}_{n-1}\right)+Q^{\prime}\left[g_{n-1}\right]\left(r_{n-1}(\cdot, \Delta \beta)\right)+R_{n-1} \text {, }
$$

where for all $a>1$,

$$
\begin{aligned}
\left\|R_{n-1}\right\|_{a} & =o\left(\Delta \beta\left\|\dot{g}_{n-1}\right\|_{c(a)}+\left\|r_{n-1}(\cdot, \Delta \beta)\right\|_{c(a)}\right) \\
& =o(\Delta \beta), \quad c(a) \stackrel{\text { def }}{=} \frac{a \delta^{\lambda}}{1+a\left(\delta^{\lambda}-1\right)} .
\end{aligned}
$$

Since the operator $Q^{\prime}\left[g_{n-1}\right]$ is linear and continuous, the function

$$
Q^{\prime}\left[g_{n-1}\right]\left(r_{n-1}(\cdot, \Delta \beta)\right)+R_{n-1}
$$

obeys the conditions imposed on $r_{n}$, thus $\dot{g}_{n}$ exists and

$$
\dot{g}_{n}=Q^{\prime}\left[g_{n-1}\right]\left(\dot{g}_{n-1}\right) .
$$

Let $g_{n}^{(k)} \stackrel{\text { def }}{=} D_{z}^{k} g_{n}, k \in \mathbb{N}$, then claim (iii) of Proposition 2.1 implies

Corollary 2.3 For every $n \in \mathbb{N}_{0}$ and $k \in \mathbb{N}$, the map $g_{n}^{(k)}: J(g) \rightarrow \mathcal{A}_{1}$ is differentiable on $\operatorname{Int} J(g)$, its derivative at $\beta$ is an entire function $\dot{g}_{n}^{(k)}$ from $\mathcal{A}_{1}$. For every $z_{0} \in \mathbb{C}, g_{n}^{(k)}\left(z_{0}, \beta\right)$ is $\beta$-differentiable on $\operatorname{Int} J(g)$ and

$$
\frac{\partial g_{n}^{(k)}\left(z_{0}, \beta\right)}{\partial \beta}=\dot{g}_{n}^{(k)}\left(z_{0}, \beta\right) .
$$




\subsection{Invariant Sets and Fixed Points}

By Lemma 2.3, for chosen $g \in \mathcal{L}^{+}$and $\tau \in I(g), \mathcal{L}_{\alpha}^{+}$is an invariant set of $T$. It turns out that this set contains a subset which $T$ maps into itself as well. Proposition 2.7 implies that such one is

$$
\mathcal{G} \stackrel{\text { def }}{=}\{f(z)=C \exp (u z) \mid C>0, u \geq 0\} \subset \mathcal{L}^{+} .
$$

In fact

$$
T(C \exp (u z))=C^{\delta}\left(1-u \tau \delta^{-\lambda}\right)^{-\theta} \exp \left(\frac{u \delta^{-\lambda} z}{1-u \tau \delta^{-\lambda}}\right),
$$

which also yields that $\mathcal{G}$ contains the following fixed points of $T$ :

$$
\begin{aligned}
& f_{i, *}(z)=C_{i, *} \exp \left(u_{i, *} z\right), \quad i=1,2 \\
& C_{1, *}=1, u_{1, *}=0 ; \quad C_{2, *}=\delta^{-\lambda \theta /(\delta-1)}, u_{2, *}=\frac{1}{\tau}\left(\delta^{\lambda}-1\right) .
\end{aligned}
$$

Consider the sequence $\left\{f_{n} \mid n \in N_{0}, f_{0}=C_{0} g=C_{0} \exp (\alpha z) \in \mathcal{G}\right\}$. Вy means of (2.31), one can calculate $f_{n}$ explicitly

$$
\begin{aligned}
& f_{n}(z)=C_{n} \exp \left(u_{n} z\right), \\
& C_{n}=C_{0}^{\delta^{n}} \Xi_{n}, \quad u_{n}=\frac{\alpha \delta^{-n \lambda}}{1-\frac{\alpha \tau}{\delta^{\lambda}-1}\left(1-\delta^{-n \lambda}\right)}, \\
& \Xi_{n}=\xi_{n} \prod_{l=1}^{n-1} \xi_{l}^{(\delta-1) \delta^{n-1-l}}, \xi_{l}=\left[1-\frac{\alpha \tau}{\delta^{\lambda}-1}\left(1-\delta^{-l \lambda}\right)\right]^{-\theta} .
\end{aligned}
$$

In this case we may check the validity of Theorem 1.2 directly. Set

$$
\begin{gathered}
\tau_{*} \stackrel{\text { def }}{=} \frac{1}{\alpha}\left(\delta^{\lambda}-1\right), \\
C(\tau) \stackrel{\text { def }}{=} \prod_{k=0}^{\infty}\left(\frac{\delta^{\lambda}-1-\alpha \tau+\alpha \tau \delta^{-(k-1) \lambda}}{\delta^{\lambda}-1-\alpha \tau+\alpha \tau \delta^{-k \lambda}}\right)^{\theta \delta^{-k-1}} .
\end{gathered}
$$

Then for $\tau<\tau_{*}$, the sequence $\left\{f_{n} \mid n \in \mathbb{N}_{0}, f_{0}(z)=C(\tau) \exp (\alpha z)\right\}$ converges in $\mathcal{A}_{\alpha}$ to $f_{1, *} \equiv 1$. If for such $\tau$, one chooses $f_{0}(z)=C_{0} \exp (\alpha z)$ with $C_{0}<C(\tau)$ (resp. $C_{0}>C(\tau)$ ), then $C_{n}$ in (2.34) tends to zero (resp. to infinity). For $\tau=\tau_{*}$, one has in (2.36) and (2.34) respectively

$$
\begin{aligned}
& C\left(\tau_{*}\right)=C_{2, *}, \\
& C_{n}=C_{0}^{\delta^{n}} \exp \left(\lambda \theta \frac{\delta^{n}-1}{\delta-1} \log \delta\right), \quad u_{n}=\alpha .
\end{aligned}
$$


Thus for all $n \in \mathbb{N}_{0}, C_{n}=C_{2, *}$ if $C_{0}=C\left(\tau_{*}\right)=C_{2, *}$. For $C_{0}<C_{2, *}$ (resp. $\left.C_{0}>C_{2, *}\right), C_{n}$ tends to zero (resp. to infinity). The fixed points of $Q$ in $\mathcal{G}$ are

$$
g_{i, *}(z)=C_{i, *} \exp \left(v_{i, *} z\right), \quad v_{1, *}=0, \quad v_{2, *}=1 .
$$

To describe the stability of the fixed points (2.32) we solve the eigenvalue problem

$$
T^{\prime}\left[f_{i, *}\right](h)=\Lambda h
$$

To this end we set

$$
h(z)=f_{i, *}(z) p(z)=C_{i, *} \exp \left(u_{i, *} z\right) p(z),
$$

with $p$ being a polynomial, and obtain from (2.20) and Proposition 2.7

$$
\begin{aligned}
T^{\prime}\left[f_{i, *}\right](h)= & \delta C_{i, *}^{\delta}\left(1-u_{i, *} \delta^{-\lambda} \tau\right)^{-\theta} \exp \left(\frac{u_{i, *} \delta^{-\lambda} z}{1-u_{i, *} \delta^{-\lambda} \tau}\right) \\
& \exp \left(\tau\left(1-u_{i, *} \delta^{-\lambda} \tau\right) \Delta_{\theta}\right) p\left(\frac{z \delta^{-1-\lambda}}{\left(1-u_{i, *} \delta^{-\lambda} \tau\right)^{2}}\right) .
\end{aligned}
$$

Suppose that $\operatorname{deg} p=k, k \in \mathbb{N}_{0}$ and apply the latter formula in (2.38). Since $\exp \left(\ldots \Delta_{\theta}\right)$ maps such $p$ into a polynomial $q$, $\operatorname{deg} q=k$, we may find $\Lambda_{k}^{(i)}$ setting the coefficients in front of the $k$-th powers of $z$ to be equal. This yields

$$
\Lambda_{k}^{(i)}=\frac{\delta^{-k \lambda-k+1}}{\left(1-u_{i, *} \delta^{-\lambda} \tau\right)^{2 k}}, \quad k \in \mathbb{N}_{0} .
$$

For both $f_{i, *}, \Lambda_{0}=\delta>1$, which corresponds to their instability with respect to the variation of the constant multiplier $C$. The rest of the eigenvalues which describe $f_{1 *}$ are $\Lambda_{k}^{(1)}=\delta^{-k \lambda-k+1}<1$. But for $f_{2, *}$, one has

$$
\Lambda_{k}^{(2)}=\delta^{k \lambda-k+1}, \quad k \in N_{0}
$$

The eigenvalues of $Q^{\prime}\left[g_{i, *}\right]$ are exactly the same as given by (2.39). For $\lambda \in(0,1 / 2), \Lambda_{2}^{(2)}<1$. This means that, in the corresponding spaces $\mathcal{A}_{a}$, $f_{2, *}, g_{2, *}$ have the stable manifolds of codim $=2$ and $f_{1, *}, g_{1, *}$ have those of codim $=1$. This fact plays an important role in proving the convergence to these fixed points. The proof will be done by showing that there exist $\beta_{*}>0$ and a function $C:\left(0, \beta_{*}\right] \rightarrow \mathbb{R}_{+}$such that all elements of the sequence $\left\{g_{n} \mid g_{0}(z)=C\left(\beta_{*}\right) g\left(\beta_{*} z\right)\right\}$ remain in the stable manifold of $g_{2, *}$ and the elements of $\left\{g_{n} \mid g_{0}(z)=C(\beta) g(\beta z), \beta<\beta_{*}\right\}$ remain in the stable manifold of $g_{1, *}$. The convergence of the corresponding sequences $\left\{f_{n}\right\}$ will be obtained as a direct corollary. 


\section{Proofs}

\subsection{Main Lemmas}

The case where the initials elements of the sequences considered are chosen in $\mathcal{G}$ has already been described, thus from now on we suppose that these functions are chosen outside of $\mathcal{G}$. We set (see (2.2), (2.3))

$$
g_{n}(z)=C_{n} \exp \left(\varphi_{n}(z)\right), \quad \tilde{g}_{n}(z)=\exp \left(\tilde{\varphi}_{n}(z)\right), \quad \varphi_{n}(0)=\tilde{\varphi}_{n}(0)=0,
$$

and for $k \in \mathbb{N}$,

$$
\varphi_{n}^{(k)} \stackrel{\text { def }}{=}\left(D^{k} \varphi_{n}\right)(0), \quad \tilde{\varphi}_{n}^{(k)} \stackrel{\text { def }}{=}\left(D^{k} \tilde{\varphi}_{n}\right)(0)=\delta^{n \lambda k} \varphi_{n}^{(k)} .
$$

As it has been shown above (Lemma 2.4 and Corollary 2.3), all $\varphi_{n}^{(1)}$ are differentiable, and hence continuous, functions of $\beta \in J(g)$. For $\beta=0$, all $\varphi_{n}^{(1)}$ are equal to zero, thus one may assume that, for every $n \in \mathbb{N} N_{0}$, the following inequality

$$
\varphi_{n}^{(1)}<\left(1-\delta^{-\lambda}\right)^{-1}
$$

holds for $\beta$ small enough, say, for $\beta \in J_{n}=\left(0, \hat{b}_{n}\right)$. Below we obtain the estimates which allow to evaluate the intervals $J_{n}$. Thus we set

$$
\nu_{n}=\frac{1}{1-\left(1-\delta^{-\lambda}\right) \varphi_{n-1}^{(1)}} ; \quad \kappa_{n}=\delta^{-\lambda} \nu_{n}
$$

Lemma 3.1 [Main estimates] For $\beta \in J_{n}$, the following estimates hold

$$
\begin{gathered}
C_{n} \geq C_{n-1}^{\delta} ; \\
C_{n} \leq C_{n-1}^{\delta} \nu_{n}^{\theta} ; \\
C_{n} \geq C_{n-1}^{\delta} \nu_{n}^{\theta} \exp \left\{\frac{1}{2} \theta(\theta+1) \kappa_{n}^{2}\left(1-\delta^{-\lambda}\right)^{2} \delta^{2 \lambda-1} \varphi_{n-1}^{(2)}\right\} .
\end{gathered}
$$

Equalities hold in (3.5)-(3.7) only in the case $\theta=0$. Further

$$
\begin{aligned}
& \varphi_{n}^{(2)}>\delta^{2 \lambda-1} \kappa_{n}^{4} \varphi_{n-1}^{(2)} ; \\
& \varphi_{n}^{(1)}<\kappa_{n} \varphi_{n-1}^{(1)} ; \\
& \varphi_{n}^{(1)}>\kappa_{n} \varphi_{n-1}^{(1)}+(\theta+1)\left(1-\delta^{-\lambda}\right) \delta^{2 \lambda-1} \kappa_{n}^{3} \hat{\varphi}_{n-1}^{(2)} ;
\end{aligned}
$$




$$
\begin{gathered}
\tilde{\varphi}_{n}^{(2)}>\delta^{-1} \nu_{n}^{4} \tilde{\varphi}_{n-1}^{(2)} ; \\
\tilde{\varphi}_{n}^{(1)}<\nu_{n} \tilde{\varphi}_{n-1}^{(1)} ; \\
\tilde{\varphi}_{n}^{(1)}>\nu_{n} \tilde{\varphi}_{n-1}^{(1)}+(\theta+1)\left(1-\delta^{-\lambda}\right) \delta^{-1} \nu_{n}^{3} \delta^{-(n-1) \lambda} \tilde{\varphi}_{n-1}^{(2)} .
\end{gathered}
$$

Proof. First we prove (3.5). Consider

$$
S(t, z) \stackrel{\text { def }}{=} \exp \left(t \Delta_{\theta}\right)\left[g_{n-1}\left(z \delta^{-1-\lambda}\right)\right]^{\delta}, t \in[0, \bar{t}], \quad \bar{t} \stackrel{\text { def }}{=} \delta^{\lambda}-1, n \in \mathbb{N} .
$$

Taking into account Corollary 2.1, (2.8), (2.22), and Lemma 2.3 one concludes that $S$ belongs to $\mathcal{L}^{+}$and satisfies the equation

$$
\frac{\partial S}{\partial t}=\Delta_{\theta} S, \quad S(0, z)=\left[g_{n-1}\left(z \delta^{-1-\lambda}\right)\right]^{\delta}, \quad S(\bar{t}, z)=g_{n}(z) .
$$

We set

$$
S_{k}(t) \stackrel{\text { def }}{=}\left(D_{z}^{k} S\right)(t, 0), \quad k \in N_{0},
$$

and obtain from (3.15) and (1.7)

$$
\frac{\partial S_{0}(t)}{\partial t}=\theta S_{1}(t)
$$

Since $S \in \mathcal{L}^{+}, S_{1}(t)>0$ and for all $t \in[0, \bar{t}]$,

$$
S_{0}(\bar{t})>S_{0}(0) \text { for } \theta>0, \quad S_{0}(\bar{t})=S_{0}(0) \text { for } \theta=0 .
$$

This estimate and the boundary conditions (3.15) gives (3.5). Now we set

$$
p_{n}(z)=\exp \left(-\varphi_{n}^{(1)} z\right) g_{n}(z)
$$

insert $g_{n-1}(z)=\exp \left(\varphi_{n-1}^{(1)} z\right) p_{n-1}(z)$ into $(2.22)$, and use (2.12). Then

$$
g_{n}(z)=\nu_{n}^{\theta} \exp \left(\kappa_{n} \varphi_{n-1}^{(1)} z\right) \exp \left(t_{n} \kappa_{n}^{-2} \Delta_{\theta}\right)\left[p_{n-1}\left(z \delta^{\lambda-1} \kappa_{n}^{2}\right)\right]^{\delta},
$$

where $t_{n}=\left(1-\delta^{-\lambda}\right) \kappa_{n}$. For $t \in\left[0, t_{n}\right]$, we define

$$
\exp R(t, z)=\exp \left(t \Delta_{\theta}\right)\left[p_{n-1}\left(z \delta^{\lambda-1}\right)\right]^{\delta} .
$$

According to Proposition 2.7, the above function can be written in the form

$$
\exp R(t, z)=\exp (\hat{u} z) \hat{p}(z)
$$


where $\hat{u}<0$ and $\hat{p}$ belongs to $\mathcal{L}^{+}$. Thus Proposition 2.3 yields for $k \geq 2$

$$
(-1)^{k-1} R_{k}(t)=(-1)^{k-1}\left(D_{z}^{k} \log \hat{p}(0)>0 .\right.
$$

Besides, we have

$$
R(0, z)=\delta \log C_{n-1}-\delta^{\lambda} \varphi_{n-1}^{(1)} z+\delta \varphi_{n-1}\left(z \delta^{\lambda-1}\right),
$$

which gives

$$
R_{0}(0)=\delta \log C_{n-1}, \quad R_{1}(0)=0, \quad R_{2}(0)=\delta^{2 \lambda-1} \varphi_{n-1}^{(2)} .
$$

Comparing (3.18) and (3.17), one obtains

$$
R\left(t_{n}, z \kappa_{n}^{2}\right)=\varphi_{n}(z)-\kappa_{n} \varphi_{n-1}^{(1)} z+\log C_{n}-\theta \log \nu_{n}
$$

which yields

$$
\begin{aligned}
& R_{0}\left(t_{n}\right)=\log C_{n}-\theta \log \nu_{n}, \quad R_{1}\left(t_{n}\right)=\kappa_{n}^{-2}\left(\varphi_{n}^{(1)}-\kappa_{n} \varphi_{n-1}^{(1)}\right), \\
& R_{2}\left(t_{n}\right)=\kappa_{n}^{-4} \varphi_{n}^{(2)} .
\end{aligned}
$$

For $R(t, z)$, we obtain from (3.18) an equation of the type of (1.9), (3.15)

$$
\frac{\partial R(t, z)}{\partial t}=\theta\left(D_{z} R\right)(t, z)+z\left[\left(D_{z}^{2} R\right)(t, z)+\left(D_{z} R\right)^{2}(t, z)\right]
$$

with the initial condition given by (3.20). It yields in turn

$$
\begin{aligned}
& \frac{\partial R_{0}(t)}{\partial t}=\theta R_{1}(t), \\
& \frac{\partial R_{1}(t)}{\partial t}=(\theta+1) R_{2}(t)+R_{1}^{2}(t), \\
& \frac{\partial R_{2}(t)}{\partial t}=(\theta+2) R_{3}(t)+4 R_{1}(t) R_{2}(t) .
\end{aligned}
$$

By the sign rule (3.19), $R_{2}(t)<0$, thus for every $t_{*}$ such that $R_{1}\left(t_{*}\right)=0$, one has from (3.25)

$$
\frac{\partial R_{1}}{\partial t}\left(t_{*}\right)<0
$$

Clearly, such $t_{*}$ is at most one. Since $R_{1}(0)=0$, one has $t_{*}=0$ and

$$
R_{1}(t)<0, \quad \forall t \in\left(0, t_{n}\right]
$$


which yields in (3.24)

$$
R_{0}\left(t_{n}\right)>R_{0}(0) \text { for } \theta>0, \quad R_{0}\left(t_{n}\right)=R_{0}(0) \text { for } \theta=0,
$$

and $R_{1}\left(t_{n}\right)<0$, implying $(3.6)$ and $(\sqrt{3.9})$ if the conditions $(\sqrt{3.20})-(3.23)$ are taken into account. Applying again (3.19) and (3.27) in (3.26) we get

$$
\frac{\partial R_{2}(t)}{\partial t}>0, \quad \forall t \in\left(0, t_{n}\right]
$$

which yields in (3.25)

$$
R_{1}(t)>t(\theta+1) R_{2}(0)
$$

and

$$
R_{2}(0)<R_{2}\left(t_{n}\right) .
$$

The latter gives (3.8). Taking in (3.29) $t=t_{n}$ one obtains (3.10). To obtain (3.7) one observes that (3.29) and (3.27) yield in (3.24) for $\theta>0$

$$
R_{0}\left(t_{n}\right)-R_{0}(0)>\frac{1}{2} t_{n}^{2} \theta(\theta+1) R_{2}(0) .
$$

For $\theta=0$, we have already obtained $R_{0}\left(t_{n}\right)=R_{0}(0)$. Finally, (3.11)-(3.13) follow directly from (3.8)-(3.10) and (3.2 ).

By the first condition in $(1.18)$, there exists $\sigma \in\left[\delta^{(2 \lambda-1) / 4}, 1\right)$ such that

$$
\frac{m_{2}(g)}{\left[\alpha+m_{1}(g)\right]^{2}}=\frac{\delta^{1 / 2}}{\theta+1} \frac{1-\sigma}{\delta^{\lambda}-\sigma} .
$$

For such $\sigma$, we set

$$
\begin{aligned}
& \Phi^{(1)} \stackrel{\text { def }}{=} \frac{1-\sigma \delta^{-\lambda}}{1-\delta^{-\lambda}}, \\
& \Phi^{(2)} \stackrel{\text { def }}{=}-\Phi^{(1)} \frac{\delta^{1-\lambda}}{\theta+1} \frac{\sigma^{2}(1-\sigma)}{\delta^{\lambda}-1} .
\end{aligned}
$$

Lemma 3.2 The following triple $\mathcal{I}_{n}=\left(i_{n}^{1} ; i_{n}^{2} ; i_{n}^{3}\right)$ of statements:

$$
\begin{aligned}
i_{n}^{1} & =\left\{\exists \beta_{n}^{+} \in J(g): \varphi_{n}^{(1)}=\Phi^{(1)}, \beta=\beta_{n}^{+} ; \quad \varphi_{n}^{(1)}<\Phi^{(1)}, \beta<\beta_{n}^{+}\right\}, \\
i_{n}^{2} & =\left\{\exists \beta_{n}^{-} \in J(g): \varphi_{n}^{(1)}=1, \beta=\beta_{n}^{-} ; \quad \varphi_{n}^{(1)}<1, \beta<\beta_{n}^{-}\right\}, \\
i_{n}^{3} & =\left\{\forall \beta \leq \beta_{n}^{+}: \varphi_{n}^{(2)} \geq \Phi^{(2)}\right\},
\end{aligned}
$$

holds true for all $n \in \mathbb{N}_{0}$. 
Proof. For $n=0$, we have $\varphi_{0}^{(1)}=\beta\left(\alpha+m_{1}(g)\right), \varphi_{0}^{(2)}=-\beta^{2} m_{2}(g)$. Thus we set

$$
\beta_{0}^{-}=\frac{1}{\alpha+m_{1}(g)}, \quad \beta_{0}^{+}=\frac{\Phi^{(1)}}{\alpha+m_{1}(g)}>\beta_{0}^{-} .
$$

First let us prove that $\beta_{0}^{+} \in J(g)$. If $\alpha=0, \beta_{0}^{+}$needs only to be finite, which obviously holds. For $\alpha>0$, the definitions (3.34) and (3.32) yield for $\beta=\beta_{0}^{+}$

$$
\varphi_{0}^{(1)}=\Phi^{(1)}=\frac{\delta^{1 / 2}}{\delta^{1 / 2}-(\theta+1) m_{2}(g) /\left[\alpha+m_{1}(g)\right]^{2}},
$$

thus

$$
\varphi_{0}^{(1)}=\frac{\delta^{1 / 2}}{\delta^{1 / 2}-(\theta+1)\left(\beta_{0}^{+}\right)^{2} m_{2}(g) /\left(\varphi_{0}^{(1)}\right)^{2}} .
$$

This equation can be solved with respect to $\varphi_{0}^{(1)}$

$$
\varphi_{0}^{(1)}=\frac{1}{2}\left\{1+\left[1+4 \delta^{-1 / 2}(\theta+1)\left(\beta_{0}^{+}\right)^{2} m_{2}(g)\right]^{1 / 2}\right\} .
$$

Hence making use of the second condition in (1.18) one gets

$$
\begin{aligned}
\beta_{0}^{+}\left(\alpha+m_{1}(g)\right) & =\varphi_{0}^{(1)}<\frac{1}{2}\left\{1+1+\left[4 \delta^{-1 / 2}(\theta+1)\left(\beta_{0}^{+}\right)^{2} m_{2}(g)\right]^{1 / 2}\right\} \\
& =1+\left[\delta^{-1 / 2}(\theta+1)\left(\beta_{0}^{+}\right)^{2} m_{2}(g)\right]^{1 / 2} \leq 1+\beta_{0}^{+} m_{1}(g) .
\end{aligned}
$$

Therefore, $\beta_{0}^{+} \in J(g)$ and $i_{0}^{1}, i_{0}^{2}$ are true. To prove $i_{0}^{3}$ we to apply ( 3.31). Indeed, for $\beta=\beta_{0}^{+}$,

$$
\begin{aligned}
\varphi_{0}^{(2)} & =-\left(\beta_{0}^{+}\right)^{2} m_{2}(g)=-\left(\Phi^{(1)}\right)^{2} \frac{m_{2}(g)}{\left[\alpha+m_{1}(g)\right]^{2}}=-\left(\Phi^{(1)}\right)^{2} \frac{\delta^{1 / 2}}{\theta+1} \frac{1-\sigma}{\delta^{\lambda}-\sigma} \\
& =\left(\Phi^{(1)}\right)^{2}\left(\Phi^{(1)}\right)^{-2} \Phi^{(2)} \delta^{\lambda-1 / 2} \sigma^{2} \geq \Phi^{(2)} .
\end{aligned}
$$

This proves $\mathcal{I}_{0}$. Note that the estimate (3.3) with $n=0$ holds for $\beta \in\left(0, \beta_{0}^{+}\right]$. To prove the implication $\mathcal{I}_{n-1} \Rightarrow \mathcal{I}_{n}$, we remark that, for $\beta=\beta_{n-1}^{+}, i_{n-1}^{1}$ yields $\varphi_{n-1}^{(1)}=\Phi^{(1)}$ and $\kappa_{n}=\sigma^{-1}$ ( see (3.4)). Now for $\varphi_{n}^{(1)}$, we have the following possibilities: (a) the estimate (3.3) holds for $\beta=\beta_{n-1}^{+}$; (b) this estimate does not hold for such $\beta$. In the case (a) one may apply Lemma 3.1. Then by means of $i_{n-1}^{3}$, (3.10), (3.32), and (obtain

$$
\varphi_{n}^{(1)}>\sigma^{-1} \Phi^{(1)}+(\theta+1)\left(1-\delta^{-\lambda}\right) \sigma^{-3} \delta^{2 \lambda-1} \Phi^{(2)}=\Phi^{(1)} .
$$


In the case (b) we simply have

$$
\varphi_{n}^{(1)} \geq \frac{1}{1-\delta^{-\lambda}} \geq \frac{1-\sigma \delta^{-\lambda}}{1-\delta^{-\lambda}}=\Phi^{(1)} .
$$

For $\beta=\beta_{n-1}^{-}$, we have $\varphi_{n-1}^{(1)}=1$ and $\kappa_{n}=1$. Therefore, $\varphi_{n}^{(1)}<1$ for $\beta \leq$ $\beta_{n-1}^{-}$, as follows from $i_{n-1}^{2}$ and (3.9). By Corollary 2.3, $\varphi_{n}^{(1)}$ is a continuous function of $\beta$, thus there exists at least one value of $\beta=\tilde{\beta}_{n}^{+} \in\left(\beta_{n-1}^{-}, \beta_{n-1}^{+}\right)$ such that $\varphi_{n}^{(1)}=\Phi^{(1)}$. The smallest such one is set to be $\beta_{n}^{+}$. The existence of $\beta_{n}^{-} \in\left(\beta_{n-1}^{-}, \beta_{n}^{+}\right)$can be established in the same way. For $\beta \leq \beta_{n}^{+}$, we have $\beta \leq \beta_{n-1}^{+}$and then $\varphi_{n-1}^{(1)} \leq \Phi^{(1)}$ due to $i_{n-1}^{1}$. This yields $\kappa_{n} \leq \sigma^{-1}$, then we get from (3.8)

$$
\varphi_{n}^{(2)}>\sigma^{-4} \delta^{2 \lambda-1} \varphi_{n-1}^{(2)} \geq \varphi_{n-1}^{(2)} \geq \Phi^{(2)},
$$

where the following estimates were used: $\varphi_{n}^{(2)}<0, \forall n \in N_{0} ; \sigma^{-1} \leq \delta^{(1-2 \lambda) / 4}$.

Corollary 3.1 The inequality (3.3) holds for $\beta \leq \beta_{n}^{+}$, thus $J_{n}$ is nonempty.

Lemma 3.3 There exists $\beta_{*} \in J(g)$ such that, for $\beta=\beta_{*}$,

$$
1<\varphi_{n}^{(1)}<\Phi^{(1)}, \forall n \in \mathbb{N}_{0} .
$$

For $\beta<\beta_{*}$, the above upper estimate also holds and, moreover, there exists $K=K(\beta)>0$, such that

$$
\varphi_{n}^{(1)}<K \delta^{-\lambda n}, \forall n \in \mathbb{N}_{0} .
$$

Proof. Consider the set $\Delta_{n} \stackrel{\text { def }}{=}\left\{\beta \in\left(0, \beta_{n}^{+}\right) \mid 1<\varphi_{n}^{(1)}<\Phi^{(1)}\right\}$. Just above we have shown that $\Delta_{n} \subseteq\left(\beta_{n}^{-}, \beta_{n}^{+}\right), \Delta_{n}$ is nonempty and open. Let us prove that $\Delta_{n} \subseteq \Delta_{n-1}$. Suppose there exists some $\beta \in \Delta_{n}$ which does not belong to $\Delta_{n-1}$. For this $\beta$, either $\varphi_{n-1}^{(1)} \leq 1$ or $\varphi_{n-1}^{(1)} \geq \Phi^{(1)}$. Hence either $\varphi_{n}^{(1)}<1$ or $\varphi_{n}^{(1)}>\Phi^{(1)}$ (it can be proved as above). This runs in counter with the supposition $\beta \in \Delta_{n}$, hence $\Delta_{n} \subseteq \Delta_{n-1}$. Now let $D_{n}$ be the closure of $\Delta_{n}$, then

$$
D_{n}=\left\{\beta \in\left[\beta_{n}^{-}, \beta_{n}^{+}\right] \mid 1 \leq \varphi_{n}^{(1)} \leq \Phi^{(1)}\right\} .
$$

$D_{n}$ is nonempty and $D_{n} \subseteq D_{n-1} \subseteq \ldots \subseteq D_{0} \subseteq J(g)$. Let $D_{*}=\bigcap_{n} D_{n}$, then $D_{*}$ is also nonempty and closed, and $D_{*} \subset J(g)$. Now let us show that, for 
every $\beta \in D_{*}$, the estimates (3.35) hold. Indeed, directly from the definition of $D_{*}$ one has

$$
1 \leq \varphi_{n}^{(1)} \leq \Phi^{(1)}, \forall n \in \mathbb{N}_{0} .
$$

Suppose $\varphi_{n}^{(1)}=1$ for some $n \in \mathbb{N}_{0}$ and $\beta \in D_{*}$, then $\varphi_{m}^{(1)}<1$ for all $m>n$ (see (3.9)). The latter means that this $\beta$ does not belong to all $D_{m}$ with $m>n$. This contradicts the supposition $\beta \in D_{*}$. The case $\varphi_{n}^{(1)}=\Phi^{(1)}$ can be excluded similarly. Set $\beta_{*}=\min D_{*}$. We have just proved that, for $\beta=\beta_{*}$, (3.35) holds, thus it remains to prove the second part of Lemma. To this end we take $\beta<\beta_{*}$. If $\varphi_{n}^{(1)}>1$ for all $n \in \mathbb{N}_{0}$, then either (3.35) holds or there exists such $n_{0}$ that $\varphi_{n_{0}}^{(1)} \geq \Phi^{(1)}$. This means either $\beta \in D_{*}$ or $\beta>\inf \beta_{n}^{+}$. Both these cases contradict the definition of $\beta_{*}$. Hence there exists $n_{0}$ such that $\varphi_{n_{0}-1}^{(1)} \leq 1$, then $\varphi_{n}^{(1)}<1$ for all $n \geq n_{0}$. In what follows, the definition (3.4) and the estimate (3.9) imply for the sequences $\left\{\varphi_{n}^{(1)}, n \geq n_{0}\right\}$ and $\left\{\kappa_{n}, n \geq n_{0}\right\}$ to be strictly decreasing. Then for all $n>n_{0}$, one has (see (3.9))

$$
\varphi_{n}^{(1)}<\kappa_{n} \varphi_{n-1}^{(1)}<\ldots<\kappa_{n} \kappa_{n-1} \ldots \kappa_{n_{0}+1} \varphi_{n_{0}}^{(1)}<\left(\kappa_{n_{0}+1}\right)^{n-n_{0}} .
$$

Since $\kappa_{n_{0}+1}<1$, one has $\sum_{n=0}^{\infty} \varphi_{n}^{(1)}<\infty$. Thus there exists $0<K_{0}<\infty$ such that

$$
\prod_{n=1}^{\infty} \nu_{n} \stackrel{\text { def }}{=} K_{0} .
$$

Finally, we apply (3.9) once again and obtain

$$
\varphi_{n}^{(1)}<\delta^{-\lambda n} \nu_{n} \nu_{n-1} \ldots \nu_{1} \varphi_{0}^{(1)}<\delta^{-\lambda n} K_{0} \varphi_{0}^{(1)} \stackrel{\text { def }}{=} K \delta^{-\lambda n}, \forall n \in N_{0} .
$$

Now we state the lemmas the proof of our theorems directly follows from. The first four lemmas describe the sequences $\left\{g_{n}\right\}$ defined by (2.22) whose elements have the form (3.1).

Lemma 3.4 For every $\theta \geq 0$ and $g \in \mathcal{L}(\lambda)$, there exists $\beta_{*} \in J(g)$ such that, (i) $\quad$ for $\beta=\beta_{*}, \lim _{n \rightarrow \infty} \varphi_{n}^{(1)}=1$ and $\lim _{n \rightarrow \infty} \varphi_{n}^{(2)}=0$;

(ii) $\operatorname{for} \beta<\beta_{*}, \lim _{n \rightarrow \infty} \varphi_{n}^{(1)}=0$ and $\lim _{n \rightarrow \infty} \varphi_{n}^{(2)}=0$.

Lemma 3.5 Let $\theta, g$ and $\beta_{*}$ be as above. Then there exists $C:\left(0, \beta_{*}\right] \rightarrow \mathbb{R}_{+}$ such that the sequence $\left\{C_{n} \mid n \in \mathbb{N}_{0}, C_{n}=g_{n}(0), C_{0}=C(\beta)\right\}$, converges to $C_{2, *}$ (resp. to $C_{1, *}$ ) given by (2.39) for $\beta=\beta_{*}$ (resp. $\left.\beta<\beta_{*}\right)$. The sequence $\left\{C_{n} \mid C_{0}>C(\beta)\right\}$ is divergent, the sequence $\left\{C_{n} \mid C_{0}<C(\beta)\right\}$ tends to zero. 
Lemma 3.6 Let $\theta, g, \beta_{*}$ and $C(\beta)$ be as above. Then for $\beta=\beta_{*}$, the sequence $\left\{g_{n} \mid n \in N_{0}, g_{0}(z)=C\left(\beta_{*}\right) g(\beta z)\right\}$ converges in $\mathcal{A}_{1}$ to $g_{2, *}(z)=$ $C_{2, *} \exp (z)$ defined by (2.37).

Lemma 3.7 Let $\theta, g, \beta_{*}$ and $C(\beta)$ be as above. Then for every $\beta<\beta_{*}$

(i) the sequence $\left\{g_{n} \mid n \in \mathbb{N}_{0}, g_{0}(z)=C(\beta) g(\beta z)\right\}$ converges in $\mathcal{A}_{1}$ to $g_{1, *}(z) \equiv 1$;

(ii) the sequence $\left\{\tilde{g}_{n} \mid n \in \mathbb{N}_{0}, \tilde{g}_{0}(z)=g(\beta z)\right\}$ defined by (2.23) converges in $\mathcal{A}_{\varphi}$ to $\tilde{g}_{*}(z)=\exp (\varphi z)$ with certain $\varphi=\varphi(\beta)>0$.

Directly from the definitions (2.15), (2.16), and (2.22) one has the following corollary of the above lemmas.

Lemma 3.8 For every $\theta \geq 0$ and $g \in \mathcal{L}(\lambda)$, there exist $\tau_{*} \in I(g)$ and a function $C:\left[0 . \tau_{*}\right] \rightarrow \mathbb{R}_{+}$, such that:

(i) for $\tau<\tau_{*}$, the sequence $\left\{f_{n} \mid n \in \mathbb{N}_{0}, f_{0}(z)=C(\tau) g(z)\right\}$ converges in $\mathcal{A}_{\beta_{*}^{-1}}$ to $f_{1, *}(z) \equiv 1$;

(ii) for $\tau=\tau_{*}$, the sequence $\left\{f_{n} \mid n \in \mathbb{N}_{0}, f_{0}(z)=C\left(\tau_{*}\right) g(z)\right\}$ defined by 2.15), 2.10, and (2.17) converges in $\mathcal{A}_{\beta_{*}^{-1}}$, $\beta_{*}=\tau_{*}\left(\delta^{\lambda}-1\right)^{-1}$ to $f_{2, *}(z)=\delta^{-\lambda \theta /(\delta-1)} \exp \left(\beta_{*}^{-1} z\right)$.

\subsection{Proof of Theorems}

Proof of Theorem 1.1. As it has already been established, the function $f_{n}(t, z)$ defined by (2.15) gives the solution of the problem (1.13) provided all $f_{m}, m=0,1, \ldots n-1$ belong to the domain of the operators $T_{t}, t \in[0,1]$. The latter fact follows from Lemma 2.3 and Proposition 2.8.

Proof of Theorem 1.2. Proposition 2.9 and Lemma 3.8 yield that, for $\tau<$ $\tau_{*}$, the sequence $\left\{f_{n}(t, z)\right\}$ converges in $\mathcal{A}_{\beta_{*}^{-1}}$ to $T_{t}\left(f_{1, *}\right)$, which may be easily calculated to be identically one. For $\tau=\tau_{*}$, one has the same convergence to $T_{t}\left(f_{2, *}\right)$, which can be calculated explicitly by means of Proposition 2.7. The proof of Theorems 1.3 and 1.4 follows directly from Theorems 1.1 and 1.2 respectively on the base of the identity (1.23).

Proof of Theorem 1.5 and Theorem 1.6. By the continuity theorem (see e.g. [2], p.27), the convergence of the sequence of the transforms (1.27) $\left\{F_{\mu_{n}}\right\}$ in a certain $\mathcal{A}_{a}^{(N)}$ implies the weak convergence of the sequence $\left\{\mu_{n}\right\}$. But for the measures defined by (1.29), the transforms (1.27) are the isotropic 
functions $F_{\mu_{n}} \in \mathcal{A}_{a}^{(N)}$ and for any of them, there exists an entire function $f_{\mu_{n}} \in \mathcal{A}_{a}$ obeying (1.22). Moreover, (1.29) implies

$$
f_{\mu_{n}}(z)=T\left(f_{\mu_{n-1}}\right)(z)\left[T\left(f_{\mu_{n-1}}\right)(0)\right]^{-1}, \quad n \in \mathbb{N} .
$$

Now if one chooses the starting element $\mu_{0}=\nu$ such that

$$
\int_{\mathbb{R}^{N}} \exp ((x, y)) \nu(d y)=g((x, x)),
$$

where $g$ is the starting element of $\left\{f_{n}\right\}$ described by Lemma 3.8, then the validity of Theorem 1.5 follows from this Lemma. The assertion regarding the variance in claim (ii) may be checked directly. Similarly, the transforms $F_{\tilde{\mu}_{n}}(1.27)$ of $\tilde{\mu}_{n}$, defined by (1.32), and the elements of the sequence $\left\{\tilde{g}_{n}\right\}$, defined by (2.23) and described by claim (ii) of Lemma 3.7, obey the relation

$$
F_{\tilde{\mu}_{n}}(\sqrt{\beta} x)=\tilde{g}_{n}((x, x)), \quad n \in \mathbb{N}, \quad F_{\tilde{\mu}_{0}}(x)=g((x, x)) .
$$

Then the validity of Theorem 1.6 follows directly from claim (ii) of Lemma 3.7.

\subsection{Proof of Lemmas}

Proof of Lemma 3.4. Consider the case $\beta=\beta_{*}$, where (3.35) holds and $\varphi_{0}^{(2)} \geq \Phi^{(2)}$ by statement $i_{0}^{3}$ of Lemma 3.2 . First we prove that $\varphi_{n}^{(2)} \rightarrow 0$. Here we have such two possibilities:

(a) $\sigma>\delta^{(2 \lambda-1) / 4}$. From (3.4) and (3.35) we obtain $\kappa_{n}<\sigma^{-1}$. Thus

$$
\delta^{2 \lambda-1} \kappa_{n}^{4}<\delta^{2 \lambda-1} \sigma^{-4}<1
$$

Applying this estimate in (3.8) one gets

$$
\left|\varphi_{n}^{(2)}\right|<\delta^{2 \lambda-1} \sigma^{-4}\left|\hat{\varphi}_{n-1}^{(2)}\right|<\ldots<\left(\delta^{2 \lambda-1} \sigma^{-4}\right)^{n}\left|\hat{\varphi}_{0}^{(2)}\right| \leq\left(\delta^{2 \lambda-1} \sigma^{-4}\right)^{n}\left|\Phi^{(2)}\right| .
$$

In view of $(3.40)$, this gives

$$
\varphi_{n}^{(2)} \rightarrow 0, \quad n \rightarrow+\infty
$$

(b) $\sigma=\delta^{(2 \lambda-1) / 4}$. In this case we have only

$$
\delta^{2 \lambda-1} \kappa_{n}^{4}<\delta^{2 \lambda-1} \sigma^{-4}=1 .
$$


Making use of (3.8) one obtains

$$
0>\varphi_{n}^{(2)}>\delta^{2 \lambda-1} \kappa_{n}^{4} \varphi_{n-1}^{(2)}>\varphi_{n-1}^{(2)}>\ldots>\varphi_{0}^{(2)} \geq \Phi^{(2)}
$$

Hence $\left\{\varphi_{n}^{(2)}\right\}$ is strictly increasing and bounded. Then it is convergent and its limit, say $\varphi^{(2)}$, obeys the condition $\varphi^{(2)}>\Phi^{(2)}$. Assume now that $\varphi^{(2)} \neq 0$. Combining (3.8) and (3.41) one obtains (recall that $\varphi_{n}^{(2)}<0$ )

$$
\frac{\varphi_{n}^{(2)}}{\varphi_{n-1}^{(2)}}<\delta^{2 \lambda-1} \kappa_{n}^{4}<1,
$$

which means $\kappa_{n} \rightarrow \delta^{(1-2 \lambda) / 4}$. The latter as well as the definitions of $\kappa_{n}$ and $\Phi^{(1)}$ immediately yield

$$
\varphi_{n}^{(1)} \rightarrow \Phi^{(1)}
$$

Passing to the limit $n \rightarrow+\infty$ in (3.10) one obtains

$$
\Phi^{(1)} \geq \delta^{(1-2 \lambda) / 4} \Phi^{(1)}+(\theta+1)\left(1-\delta^{-\lambda}\right) \delta^{2 \lambda-1} \delta^{3(1-2 \lambda) / 4} \varphi^{(2)},
$$

which yields in turn

$$
\varphi^{(2)} \leq-\Phi^{(1)} \frac{(1-\sigma) \sigma^{2} \delta^{1-\lambda}}{(\theta+1)\left(\delta^{\lambda}-1\right)}=\Phi^{(2)} .
$$

The latter gives the following contradictory inequalities

$$
\Phi^{(2)}<\varphi^{(2)} \leq \Phi^{(2)} .
$$

Thus $\varphi^{(2)}=0$. To show that $\varphi_{n}^{(1)} \rightarrow 1$, we set

$$
b_{n}=(\theta+1)\left(1-\delta^{-\lambda}\right) \delta^{2 \lambda-1} \kappa_{n}^{3} \varphi_{n-1}^{(2)} .
$$

Since $\left\{\kappa_{n}\right\}$ is bounded and $\varphi_{n}^{(2)} \rightarrow 0$, one has $b_{n} \rightarrow 0$. By the estimate ( 3.35), $\left\{\varphi_{n}^{(1)}\right\}$ is bounded. Then it contains a subsequence $\left\{\varphi_{n_{i}}^{(1)}\right\}$ convergent to a certain $a \in\left[1, \Phi^{(1)}\right]$. From (3.9) and (3.10) one has

$$
0>\varphi_{n}^{(1)}-\kappa_{n} \varphi_{n-1}^{(1)}>b_{n}
$$

which yields

$$
\lim _{i \rightarrow \infty}\left(\varphi_{n_{i}}^{(1)}-\kappa_{n_{i}} \varphi_{n_{i}-1}^{(1)}\right)=0
$$


The latter can be rewritten as (see 3.4)

$$
a-\frac{\delta^{-\lambda} a}{1-\left(1-\delta^{-\lambda}\right) a}=0 .
$$

Since $\lambda>0$, the above equation has only one solution on $\left[1, \Phi^{(1)}\right]$, it is $a=1$. In what follows, the bounded sequence $\left\{\varphi_{n}^{(1)}\right\}$ has only one accumulation point, hence it converges to $a=1$ itself. In the case $\beta<\beta_{*}$ the estimate (3.36) yields $\varphi_{n}^{(1)} \rightarrow 0$. Then $\kappa_{n}$ given by (3.4) tends to $\delta^{-\lambda}$ which immediately gives in (3.8) $\varphi_{n}^{(2)} \rightarrow 0$.

Proof of Lemma 3.5. From the definitions (2.22) and (3.1) one obtains

$$
\begin{aligned}
C_{n} & =C_{n-1}^{\delta} \Psi_{n-1}(\beta), \\
\Psi_{n}(\beta) & \stackrel{\text { def }}{=}\left\{\exp \left(\left(\delta^{\lambda}-1\right) \Delta_{\theta}\right) \exp \left(\delta \varphi_{n}\left(\delta^{-1-\lambda} \cdot\right)\right)\right\}(0) .
\end{aligned}
$$

For $\theta=0, \Psi_{n}(\beta)=1$ (see Remark 2.2) and the situation with $C_{n}$ is obvious. Consider the case $\theta>0$. Then

$$
C_{n}=C_{0}^{\delta^{n}} \Xi_{n}(\beta), \quad \Xi_{n}(\beta) \stackrel{\text { def }}{=} \Psi_{n-1}(\beta) \Psi_{n-2}^{\delta}(\beta) \ldots \Psi_{0}^{\delta^{n-1}}(\beta) .
$$

Now we put $C_{0}=\zeta>0$, then $C_{n}=C_{n}(\zeta, \beta)$. By the above representation, for every fixed $\beta>0, C_{n}$ is a monotone convex differentiable function of $\zeta$ and

$$
C_{n}(\zeta, \beta)=\zeta^{\delta^{n}} \Xi_{n}(\beta), \quad \frac{\partial C_{n}}{\partial \zeta}=\delta^{n} \zeta^{-1} C_{n} .
$$

By Lemma 3.3, $\varphi_{n}^{(1)}<\Phi^{(1)}$ for all $n \in N_{0}$ and $\beta \in\left(0, \beta_{*}\right]$. This gives in (3.4) $\kappa_{n}<\sigma^{-1} \leq \delta^{(1-2 \lambda) / 4}$ for such $\beta$ and $n$. We set

$$
\begin{aligned}
\zeta^{-} & =\delta^{-\theta(1+2 \lambda) / 4(\delta-1)}, \\
\Upsilon & =\left[\zeta^{-}, 1\right] \subset \mathbb{R}_{+} .
\end{aligned}
$$

For a fixed $\beta \in\left(0, \beta_{*}\right]$, let us prove that the following inductive statements hold true for all $n \in \mathbb{N}_{0}$

$$
\begin{aligned}
& i_{n}^{+}=\left\{\exists \zeta_{n}^{+} \in \Upsilon: C_{n}\left(\zeta_{n}^{+}, \beta\right)=1\right\}, \\
& i_{n}^{-}=\left\{\exists \zeta_{n}^{-} \in \Upsilon: C_{n}\left(\zeta_{n}^{-}, \beta\right)=\zeta^{-}\right\} .
\end{aligned}
$$

Since $C_{n}$ is a monotone convex function of $\zeta$ (3.44), such $\zeta_{n}^{ \pm}$are unique. We set $\zeta_{0}^{+}=1, \zeta_{0}^{-}=\zeta^{-}$. Then $C_{0}=\zeta$ obeys the above conditions, thus $i_{0}^{ \pm}$are true. Now suppose that $i_{n-1}^{ \pm}$are true. Then (3.5) and (3.6) yield for $\theta>0$

$$
C_{n}\left(\zeta_{n-1}^{+}, \beta\right)>1, \quad C_{n}\left(\zeta_{n-1}^{-}, \beta\right)<\zeta^{-} .
$$


Taking into account that $C_{n}$ depends on $\zeta$ as given by (3.44) one concludes that there exist $\zeta_{n}^{ \pm}$such that

$$
\zeta_{n-1}^{-}<\zeta_{n}^{-}<\zeta_{n}^{+}<\zeta_{n-1}^{+}
$$

and the statements $i_{n}^{ \pm}$hold true. Set

$$
\Upsilon_{n}=\left[\zeta_{n}^{-}, \zeta_{n}^{+}\right]
$$

Then

$$
\Upsilon_{n} \subset \Upsilon_{n-1} \subset \ldots \subset \Upsilon
$$

and there exists $\tilde{\zeta}_{n} \in \Upsilon_{n}$ such that

$$
\zeta_{n}^{+}-\zeta_{n}^{-}=\left(1-\zeta^{-}\right)\left[\frac{\partial C_{n}\left(\tilde{\zeta}_{n}, \beta\right)}{\partial \zeta}\right]^{-1}
$$

Let

$$
\Upsilon^{*}=\bigcap_{n \in N_{0}} \Upsilon_{n}
$$

which is closed and nonempty. For $\zeta \in \Upsilon^{*}$, all $C_{n}$ belong to $\Upsilon$. Hence the sequence $\left\{C_{n}\right\}$ is separated from zero for such $\zeta$. This yields that the derivative given by (3.44) tends to $+\infty$ when $n \rightarrow \infty$. Taking into account all these facts one concludes

$$
\Upsilon^{*}=\left\{\zeta^{*}\right\}, \quad \zeta^{*} \in \Upsilon
$$

and, for all $n \in \mathbb{N}_{0}$,

$$
C_{n}\left(\zeta^{*}, \beta\right) \in \Upsilon
$$

It should be pointed out that $\zeta^{*}=\zeta^{*}(\beta)$. Choose $\zeta=\zeta^{*}$. Then by (3.51), the sequence $\left\{C_{n}\right\}$ is bounded, hence it contains a convergent subsequence. For $\beta=\beta_{*}$, by means of (3.6) and (3.7) one may show that such a subsequence converges to $C_{2, *}=\delta^{-\lambda \theta /(\delta-1)}$. As in the case of $\left\{\varphi_{n}^{(1)}\right\}$ considered above, this fact implies the convergence of the whole sequence to this limit. For $\beta<\beta_{*}$, one employs (3.5) and (3.6) and shows similarly the convergence of $\left\{C_{n}\right\}$ to $C_{1, *}=1$. Thus we choose the function $C(\beta)$ to be $C(\beta)=\zeta^{*}(\beta)$.

Proof of Lemma 3.6. It follows from Lemmas 2.1, 3.4, and 3.5.

Proof of Lemma 3.7. Claim (i) follows from the lemmas just mentioned. To prove claim (ii) we fix $\beta<\beta_{*}$ and show the convergence of $\left\{\tilde{\varphi}_{n}^{(2)}\right\}$ to zero. Indeed, (3.11) and (3.38) imply

$$
\left|\tilde{\varphi}_{n}^{(2)}\right|<\delta^{-n}\left(\nu_{n} \nu_{n-1} \ldots \nu_{1}\right)^{4}\left|\tilde{\varphi}_{0}^{(2)}\right|<\delta^{-n} K_{0}^{4}\left|\tilde{\varphi}_{0}^{(2)}\right| \text {. }
$$


Thus to complete the proof we have only to show that $\left\{\tilde{\varphi}_{n}^{(1)}\right\}$ is a Cauchy sequence. To this end for $n \in \mathbb{N}$ and $p \in \mathbb{N}$, we set

$$
\begin{gathered}
a_{n, p}=\nu_{n+p} \nu_{n+p-1} \ldots \nu_{n+1} \\
b_{n, p}=(\theta+1)\left(1-\delta^{-\lambda}\right) \delta^{-1} \sum_{s=n}^{n+p-1} \nu_{n+p} \nu_{n+p-1} \ldots \nu_{s+2} \nu_{s+1}^{3} \delta^{-\lambda s} \tilde{\varphi}_{s}^{(2)} .
\end{gathered}
$$

Then the convergence of the product (3.38) yields

$$
a_{n, p}-1<\left(\prod_{k=n+1}^{\infty} \nu_{k}\right)-1 \rightarrow 0, \quad n \rightarrow+\infty .
$$

On the other hand, (3.38) and (3.52) give

$$
\left|b_{n, p}\right|<(\theta+1)\left(1-\delta^{-\lambda}\right) \delta^{-1} K_{0}^{7}\left|\tilde{\varphi}_{0}^{(2)}\right| \sum_{s=n}^{\infty} \delta^{-(1+\lambda) s} \rightarrow 0, \quad n \rightarrow+\infty .
$$

The estimates (3.12) and (3.13) yield respectively

$$
\tilde{\varphi}_{n+p}^{(1)}<a_{n, p} \tilde{\varphi}_{n}^{(1)}, \quad \tilde{\varphi}_{n+p}^{(1)}>a_{n, p} \tilde{\varphi}_{n}^{(1)}+b_{n, p} .
$$

Therefore

$$
\left(a_{n, p}-1\right) \tilde{\varphi}_{n}^{(1)}+b_{n, p}<\tilde{\varphi}_{n+p}^{(1)}-\tilde{\varphi}_{n}^{(1)}<\left(a_{n, p}-1\right) \tilde{\varphi}_{n}^{(1)} .
$$

Having in mind (3.2) and (3.36), one gets

$$
0<\tilde{\varphi}_{n}^{(1)}=\delta^{\lambda n} \varphi_{n}^{(1)}<K
$$

Now it suffices to apply the latter estimate together with (3.55) and (3.56) in $(3.58)$ and conclude that $\left\{\tilde{\varphi}_{n}^{(1)}\right\}$ is a Cauchy sequence. Thus, for every $\beta<\beta_{*}$, there exists $\tilde{\varphi}=\tilde{\varphi}(\beta)>0$ such that $\tilde{\varphi}_{n}^{(1)} \rightarrow \tilde{\varphi}$. Now we apply Lemma 2.1 and obtain the convergence to be proved.

Remark 3.1 When proving the convergence of $\left\{\tilde{\varphi}_{n}^{(1)}\right\}$, the limit of this sequence has been estimated. Namely, we have obtained (see (3.59))

$$
\lim _{n \rightarrow \infty} \tilde{\varphi}_{n}^{(1)} \leq K=\varphi_{0}^{(1)} \prod_{n=1}^{\infty} \nu_{n}=\prod_{n=1}^{\infty} \frac{\hat{\varphi}_{0}^{(1)}}{1-\left(1-\delta^{-\lambda}\right) \varphi_{n-1}^{(1)}} .
$$

This bound is achieved for $f_{0}(z)=C \exp (\alpha z)$ (in this case we may calculate $\tilde{g}_{n}$ explicitly, see (2.34)). It is quite likely that this bound is achieved also in the general case, but to prove this conjecture we would need more sophisticated estimates than (3.19) or (3.10). 


\section{References}

[1] V.N. Denisov, On Stabilization of solutions of the Cauchy problem for parabolic equations, Nonlinear Anal.: Theory, Methods, Appl., 30 (1997) $123-127$

[2] I.A Ibragimov, Yu.V. Linnik, Independent and Stationary Sequences of Variables Random, Wolters-Noordhoff, Groningen, 1971

[3] L.Iliev, Laguerre Entire Functions, Bulgarian Academy of Sciences, Sofia, 1987

[4] S. Kamin (Kamenomostovskaya), On stabilization of solutions of the Cauchy problem for parabolic equation, Proceed. Royal Soc. Edinburgh, 76A (1977) 43-53

[5] Yu.V. Kozitsky, Hierarchical ferromagnetic vector spin model possessing the Lee-Yang property. Thermodynamic limit at the critical point and above, Journal of Statistical Physics, 87 (1997) 799-820

[6] Yu. Kozitsky, L. Wołowski, Laguerre entire functions and related locally convex spaces. Los Alamos Electronic Preprint CV/9812111, 1998

[7] B.J. Levin, Distribution of Zeros of Entire Functions. Amer. Math. Soc. 1964

[8] D. Luna, Fonctions différentiables invariantes sous l'opération d'un groupe réductif, Ann. Inst. Fourier, Grenoble, 26 (1976) 33-49.

[9] H. Weyl, The Classical Groups their Invariants and Representations, Princeton, 1938 